\title{
THE EARLY MIOCENE BATS (CHIROPTERA, MAMMALIA) FROM THE KARSTIC SITES OF ERKERTSHOFEN AND PETERSBUCH 2 (SOUTHERN GERMANY)
}

\author{
VALENTINA V. ROSINA ${ }^{1, *}$, MICHAEL RUMMEL ${ }^{2}$ \\ ${ }_{1}^{1}$ Borissiak Paleontological Institute RAS, Profsouznaya str. 123,117997 Moscow, Russia; e-mail: rossina@mail.ru. \\ ${ }^{2}$ The Natural Museum of city of Augsburg, Im Thäle 3, D - 86152 Augsburg, Germany; e-mail: Michael.Rummel@augsburg.de. \\ * corresponding author
}

Rosina, V. V., Rummel, M. (2019): The early Miocene Bats (Chiroptera, Mammalia) from the karstic sites of Erkertshofen and Petersbuch 2 (southern Germany). - Fossil Imprint, 75(3-4): 412-437, Praha. ISSN 2533-4050 (print), ISSN 2533-4069 (online).

\begin{abstract}
Fossil bats are described from the karstic deposits of the Erkertshofen 1, Erkertshofen 2 and Petersbuch 2 sites in eastern Bavaria, southern Germany (MN 4). Fossils are discussed with regard to taxonomic, stratigraphic and palaeoecologic significance. The rich fossil material represents at least 12 different bat species belonging to Megadermatidae, Rhinolophidae and Vespertilionidae. The syntopic appearance of four different rhinolophids is demonstrated for the first time for the Neogene bat assemblages of Europe. The remains of Rhinolophidae and Vespertilionidae are the most numerous, of which the proportion of typically early Miocene species Rhinolophus aff. lemanensis, R. dehmi, Hanakia agadjaniani and Miostrellus cf. petersbuchensis are significant. However, there are also remains of $R$. cf. delphinensis, M. cf. noctuloides, Plecotus cf. atavus and H. aff. antiquus, which are characteristic of the younger middle Miocene faunas of Central Europe. Analysis of the composition of the bat fauna has allowed biostratigraphic correlation of the studied faunas to be estimated at a number of other early Miocene localities in Europe.
\end{abstract}

Key words: Chiroptera, Rhinolophidae, Vespertilionidae, Megadermatidae, early Miocene, Erkertshofen, Petersbuch, Karst, southern Germany

Received: June 18, 2019 | Accepted: September 16, 2019 | Issued: December 30, 2019

\section{Introduction}

In comparison to numerous sites from the South German Molasse, the Tertiary karst sites from the Swabian and Franconian Jura offer a good opportunity to study extensive well preserved fossil assemblages of vertebrate faunas. Such karst fissure fillings often yielded large numbers of fossils of specific animal groups that were well-adapted to living in karstic cavities. Bats in particular are typical faunal elements of fissure fillings, the remains of which are sometime very abundant in the karstic oryctocenoses.

Tertiary sites with rich bat faunas are already known from southern Germany and especially from the Petersbuch site near the town of Eichstätt, Bavaria. Very diverse and abundant fauna of bats were discovered in the middle Miocene karstic sites of Petersbuch 6, 10, 18 and 31 by Ziegler (2003; $\mathrm{MN} 7 / 8$ ). Even richer bat assemblages that included different molossid and vespertilionid bats were found in other middle Miocene mammalian faunas from the freshwater travertine of the Goldberg and Steinberg localities in the southeastern part of the Nördlinger Ries (MN 6, Bavaria; Rachl 1983). Early Miocene sites with bats are also known in southern Germany. Thus, the early Miocene sites of Wintershof-West (MN 3; Ziegler 1993) and Petersbuch 28 and Petersbuch 62
(MN 3/4; Rosina and Rummel 2012) yielded very diverse and abundant bat faunas. A few unusual bat assemblages were described recently from the early Miocene deposits of the Upper Freshwater Molasse of the Forsthart and Rembach sites in eastern Bavaria, southern Germany (MN 4; Rosina and Rummel 2017). The Upper Freshwater Molasse formation is of the greatest significance for the early Miocene stratigraphy of Central Europe and the palaeoenvironmental studies of that period. The present paper provides a detailed description of the abundant fossil bat remains from the other early Miocene sites of Petersbuch 2, Erkertshofen 1 and Erkertshofen 2, which stratigraphically correlate with the Forsthart and Rembach sites of the Upper Freshwater Molasse formation and also to the MN 4 zone of the mammalian biochronological scale for the European Neogene (Steininger 1999), but have a karstic origin. The presence of bat remains at the Petersbuch 2, Erkertshofen 1 and Erkertshofen 2 sites was established earlier by R. Ziegler, to whom we are grateful for the preliminary sorting of the fossil material. The present study provides a detailed analysis of the bat assemblages from Petersbuch 2, Erkertshofen 1 and Erkertshofen 2 sites, and discusses their possible biostratigraphic implications and significance for further study of the early history of European bat fauna. 


\section{Geological setting and biostratigraphic consideration}

The karst sites of Petersbuch 2, Erkertshofen 1 and Erkertshofen 2 were discovered and exploited in 1962, 1974 and 1977 respectively and extensive fossil finds from them were included in a multitude of scientific publications (e.g. Fahlbusch 1966, Heissig 1978, Fahlbusch and Ziegler 1986, Roth 1989). The text below provides a brief description of the geological location and setting of the sites taken from the abovementioned references.

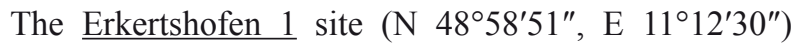
was situated in a small quarry composed of the thick-bank limestones of the White Jura Delta ("Treuchtlinger Marmor", Mittel-Kimmeridge; Heissig 1978) about $1 \mathrm{~km}$ west of Erkertshofen village and about $10 \mathrm{~km}$ north of Eichstätt. In 1962 on the west side of the $15 \mathrm{~m}$ deep quarry, a clay-filled karst fissure was cut vertically from the surface, up to 1.5 $\mathrm{m}$ wide, which split into several narrow passages towards the base. While the upper 12-13 $\mathrm{m}$ of the karst loam were devoid of fossils, very rich areas of small bone splinters were observed in the fissures near and at the base of the fracture. The material was an evenly coloured yellow-brown, fat clay with a low content of quartz sand and bean ore nodules.

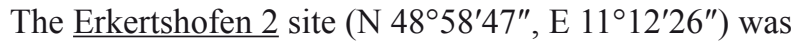
located in the same outcrop as Erkertshofen 1 (according to Fahlbusch and Ziegler 1986). In 1974/75 the site of Erkertshofen 2 represented a system of fissures, opening into the quarry floor and yielded the majority of fossils. The main fissure with fossils contained yellow-brown clay with manganese inclusions (Heissig 1978).

The Petersbuch 2 site (N 48 $58^{\prime} 39^{\prime \prime}$, E $\left.11^{\circ} 11^{\prime} 53^{\prime \prime}\right)$ was located in the "Volkert quarry" in the White Jurassic Delta. The first investigation took place in March 1977. The site consisted of a fissure system in the eastern part of the quarry. The fossil-bearing fissure system extended to about $3 \mathrm{~m}$ above the quarry floor. It contained a reddish-brown clay with iron oxide nodules and fragments of shell-like phosphate nodules. Pebbly weathering relicts from the White Jurassic were frequent. The fossils are also partly phosphatized.

According to the publications of Fahlbusch and Ziegler (1986) and Heizmann (1983), the Erkertshofen 1 and Erkertshofen 2 sites correspond to the MN $4 \mathrm{~b}$ zone of the mammalian biochronological scale for the European Neogene (Steininger 1999) and belong to the OSM A zone of the Molasse development (Kälin and Kempf 2009, Abdul Aziz et al. 2010). The Petersbuch 2 site is older than both the Erkertshofen 1 and 2 sites and corresponds to the MN 4a zone (Fahlbusch and Ziegler 1986: 51, fig. 31).

\section{Material and methods}

The 806 fossil bat specimens from Petersbuch 2, Erkertshofen 1 and Erkertshofen 2 were examined (Tab. 9). The fossil material under study is mostly represented by dentary and maxillary fragments and isolated teeth. The dental terminology follows Miller (1907); for the upper canines we follow the terminology used by Rosina (2015). The tribe taxonomy here follows Simmons (2005).

The specimens were measured in a standard way using a binocular microscope MBS-10 with ocular micrometer. The measurements are given in millimeters with $0.01 \mathrm{~mm}$ precision. Lengths of the individual teeth and tooth-rows were taken as the maximal distances between the posterior and anterior crown edges of the respective teeth. The upper teeth are indicated in upper case letters, and lower teeth are in lower letters.

The following measurements were taken:

$\mathrm{p} 4$ - the maximal length $(\mathrm{L}) \times$ width $(\mathrm{W})$ of the crown, in the case of P2, p2 and p3 - the measurements of the alveoli of the crowns;

$\mathrm{m} 1,2,3$ - length $(\mathrm{L}) \times$ width of the molar trigonid $(\mathrm{Wtr}) \times$ width of the molar talonid $(\mathrm{Wtl})$;

M1, 2, 3 - length $(\mathrm{L}) \times$ width $(\mathrm{W})$ of the crowns;

Lc-m3, Lc-m1, Lm1-3, Lm1-2, Lm2-3 - respectively the lengths of the corresponding tooth-row fragments;

Hmdm1 - the height of a mandibular corpus measured from the lingual side below $\mathrm{m} 1$;

Hmdm3 - the height of a mandibular corpus measured from the lingual side below $\mathrm{m} 3$.

The majority of the fossil material is stored in the Bavarian State Collection for Paleontology and Geology, Munich (abbreviated BSP and SNSB-BSPG). Some fossil bat specimens studied from Petersbuch 2 were from the private collection of Dr. M. Rummel (Weißenburg, Germany, abbreviated PCMRCh).

The photographs were taken using a SEM (scanning electron microscope) at AMU (Applied Materials Laboratory, University of Augsburg, Germany).

\section{Abbreviations}

coll. collection

mnd mandible

mxl maxille

sup. superior

$\mathrm{Ch} / \mathrm{G}$ coll. of fossil Chiroptera of the Palaeontological Museum of National Museum of Natural History, National Academy of Sciences of Ukraine, Kiev, Ukraine

NMA The Natural Museum of city of Augsburg, Bavaria, Germany

SMNS State Museum of Natural History Stuttgart, Germany

ZMMU Zoological Museum of Moscow State University, Moscow, Russia

\author{
Abbreviations of the biometric parameters in the tables \\ $\mathrm{m}$ arithmetic mean \\ $n$ number of specimens \\ $\mathrm{R}$ range of measurements, i.e. the difference between \\ maximum and minimum values \\ S standard deviation
}

\section{Systematic palaeontology}

Order Chiroptera BlumenBaCH, 1779
Family Megadermatidae AlLEN, 1864

Genus Megaderma É. Geoffroy SAINT-Hilaire, 1810

Megaderma franconica ZIEGLER, 1993

Text-fig. 1

M a te r i a 1. Erkertshofen 1: BSP 1962 XIX 4148, left m2; BSP 1962 XIX 4147, left mnd with m1-3; BSP 1962 XIX 4149, left M1. 
Petersbuch 2: BSP 1976 XXII 4141-4148 (8 maxillary fragments with teeth); BSP 1976 XXII 4149-4154, 4156-4158, 4165, BSP 1976 XXII 11072, PCMRCh112 (15 isolated upper teeth); BSP 1976 XXII 4110-4140, BSP 1976 XXII 11069, PCMRCh110 (33 mandibles with teeth); BSP 1976XXII 4155, 4159-4164, PCMRCh111, 113 (13 isolated lower teeth).

Measurements. See Tab. 1.

Description and comparis on s. Jaw fragments are represented by the maxillary and mandibular bones with cheek teeth and isolated teeth. They bear all the morphological traits typical of $M$. franconica as described in detail earlier (Rosina and Rummel 2012: 465-466). The fossil species of Megaderma are quite uniform in their odontology, but often differ in size (Tab. 2), and, in particular, in the degree of $\mathrm{m} 3$ talonid reduction. Both the nominative form $M$. franconica from Wintershof-West (MN 3, Germany; Ziegler 1993) and $M$. cf. franconica from Stubersheim 3 (MN 3, Germany;
Ziegler 1994) are somewhat larger and have a less reduced m3 talonid than Megaderma from Petersbuch 2 and Erkertshofen 1 (Tab. 2). The early Miocene M. brailloni SIGÉ, 1968 from Bouzigues (MN 2, France) and M. gaillardi (Trouessart, 1898) from La Grive 7 (MN 7, France), Beni Mellal (MN 7, Moroco) and Escobosa de Calatanazor (MN 6, Spain) are bigger than Megaderma from Petersbuch 2 and Erkertshofen 1 (Tab. 2; see also Sigé 1968, 1976). The middle Miocene M. lugdunensis (DEPÉRET, 1892) (MN 6, Steinberg, Goldberg, Germany and Devínská Nová Ves, Slovak Republic, and MN 5, Vieux Collonges, France), is slightly smaller and shows a less reduced $\mathrm{m} 3$ talonid than Megaderma studied from Petersbuch 2 and Erkertshofen 1 (Tab. 2, Textfig. 1b, d1; Rachl 1983: 153, fig. 53). The late Miocene M. jaegeri SigÉ, 1976 from Beni Mellal (MN 5 or MN 6, Africa; Sigé 1976) is considerably smaller than the fossils from Petersbuch 2 and Erkertshofen 1 (Tab. 2). The fossil Megaderma from Petersbuch 2 and Erkertshofen 1 (Text-fig. 1) is

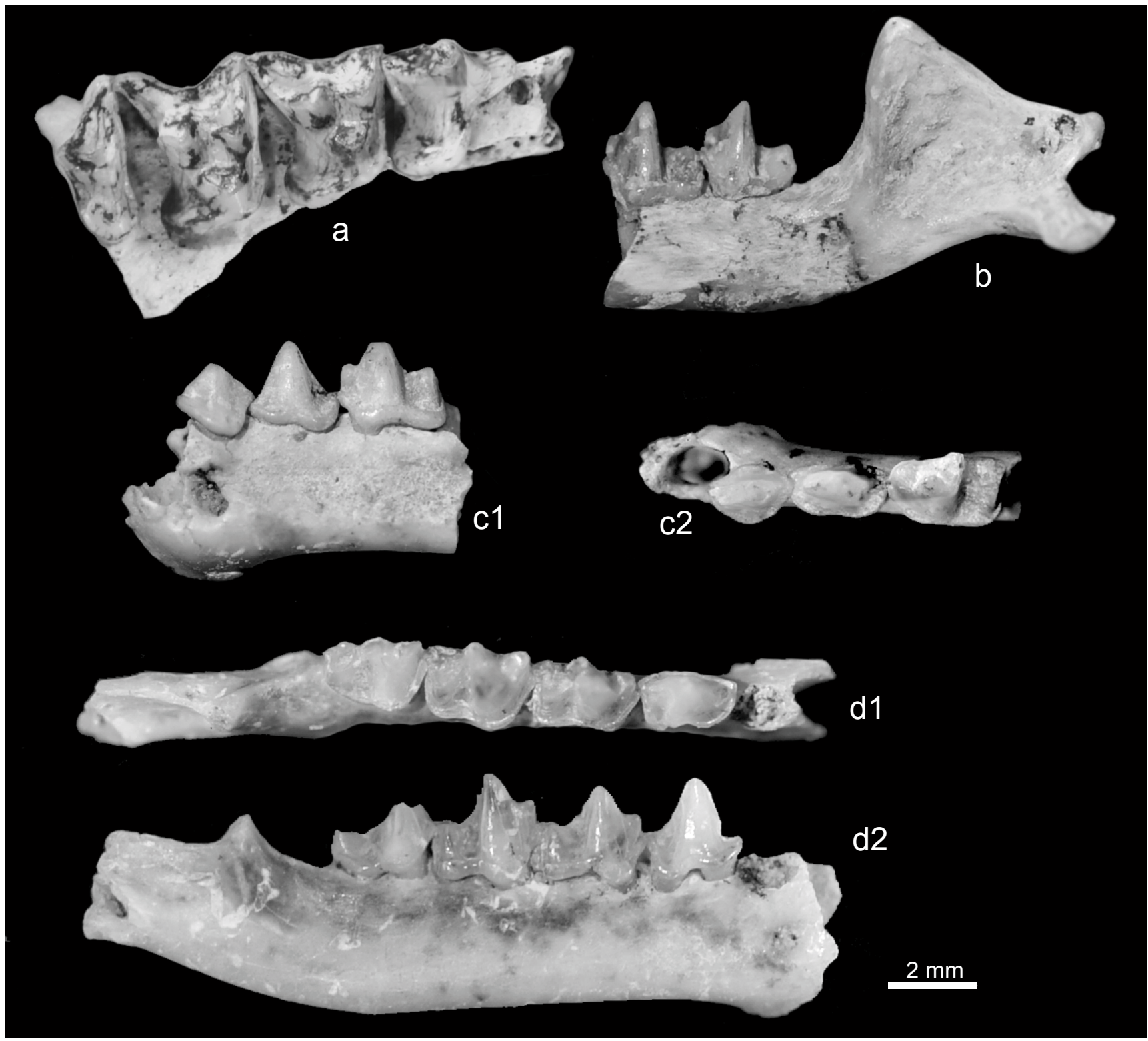

Text-fig. 1. Megaderma franconica, Petersbuch 2: a - BPS 1976 XXII 4147, right mxl with P4-M3, ventral view; b - BSP 1976 XXII 4112, left mnd with m2-3, lateral view; c - BSP 1976 XXII 4124, left mnd with p2-m1, (c1) lateral view, (c2) occlusal view; d - BSP 1976 XXII 4110, right mnd with p4-m3, (d1) occlusal view, (d2) lateral view. 
Table 1. Megaderma franconica from Petersbuch 2 and Erkertshofen 1, sample statistics of the teeth and jaws (in mm).

\begin{tabular}{|c|c|c|c|c|c|c|c|c|}
\hline \multirow{2}{*}{ Measur. } & \multicolumn{4}{|c|}{ Petersbuch 2} & \multicolumn{4}{|c|}{ Erkertshofen 1} \\
\hline & $\mathbf{n}$ & $\mathbf{R}$ & $\mathbf{m}$ & $\mathbf{S}$ & $\mathbf{n}$ & $\mathbf{R}$ & $\mathbf{m}$ & $\mathbf{S}$ \\
\hline LC & 9 & $3.15-3.35$ & 3.27 & 0.075 & - & - & - & - \\
\hline WC & 9 & $1.90-2.05$ & 1.96 & 0.049 & - & - & - & - \\
\hline $\mathrm{HC}$ & 9 & $4.40-4.90$ & 4.69 & 0.147 & - & - & - & - \\
\hline LP2 & 1 & 0.55 & - & - & - & - & - & - \\
\hline WP2 & 1 & 0.45 & - & - & - & - & - & - \\
\hline LP2 al. & 6 & $0.40-0.60$ & 0.52 & 0.082 & - & - & - & - \\
\hline WP2 al. & 6 & $0.45-0.65$ & 0.54 & 0.08 & - & - & - & - \\
\hline LP4 & 6 & $2.65-2.95$ & 2.82 & 0.103 & - & - & - & - \\
\hline WP4 & 5 & $2.30-2.50$ & 2.40 & 0.079 & - & - & - & - \\
\hline LP4-M3 & 1 & 9.15 & - & - & - & - & - & - \\
\hline LM1-M3 & 1 & 6.55 & - & - & - & - & - & - \\
\hline LM1 & 8 & $2.80-2.90$ & 2.84 & 0.042 & - & - & - & - \\
\hline WM1 & 7 & $3.00-3.20$ & 3.10 & 0.076 & - & - & - & - \\
\hline LM2 & 4 & $2.60-2.95$ & 2.79 & 0.165 & 1 & 2.75 & - & - \\
\hline WM2 & 4 & $3.20-3.50$ & 3.35 & 0.129 & 1 & 3.15 & - & - \\
\hline LM3 & 3 & $1.50-1.55$ & 1.52 & 0.029 & - & - & - & - \\
\hline WM3 & 2 & 3.20 & 3.20 & - & - & - & - & \\
\hline Lc & 5 & $2.25-2.45$ & 2.33 & 0.076 & - & - & - & - \\
\hline Wc & 5 & $1.95-2.00$ & 1.97 & 0.027 & - & - & - & - \\
\hline Hc & 5 & $4.20-4.70$ & 4.52 & 0.192 & - & - & - & - \\
\hline Lp2-p4 & 1 & 4.45 & - & - & - & - & - & - \\
\hline Lp4-m3 & 1 & 10.20 & - & - & - & - & - & - \\
\hline Lp2 & 1 & 2.10 & - & - & - & - & - & - \\
\hline Wp2 & 1 & 1.50 & - & - & - & - & - & - \\
\hline Lp4 & 4 & $2.15-2.55$ & 2.40 & 0.173 & - & - & - & - \\
\hline Wp4 & 5 & $1.20-1.45$ & 1.37 & 0.097 & - & - & - & - \\
\hline Lm1-2 & 10 & $5.15-5.75$ & 5.41 & 0.157 & 1 & 5.65 & - & - \\
\hline Lm2-3 & 10 & $4.95-5.65$ & 5.43 & 0.209 & 1 & 5.55 & - & - \\
\hline Lm1-3 & 5 & $7.75-8.20$ & 8.03 & 0.186 & 1 & 8.15 & - & - \\
\hline Lm3 & 16 & $2.60-2.90$ & 2.77 & 0.08 & 1 & 2.80 & - & - \\
\hline Wtrm3 & 17 & $1.45-1.75$ & 1.61 & 0.088 & 1 & 1.65 & - & - \\
\hline Wtlm3 & 16 & $1.00-1.20$ & 1.10 & 0.083 & 1 & 1.00 & - & - \\
\hline Lm2 & 23 & $2.60-3.15$ & 2.87 & 0.119 & 2 & 2.95 & 2.95 & - \\
\hline Wtrm2 & 23 & $1.50-1.85$ & 1.68 & 0.077 & 2 & $1.55-1.75$ & 1.65 & 0.141 \\
\hline Wtlm2 & 23 & $1.40-1.80$ & 1.60 & 0.11 & 2 & $1.60-1.80$ & 1.70 & 0.141 \\
\hline Lm1 & 15 & $2.55-2.90$ & 2.74 & 0.104 & 1 & ca. 2.80 & - & - \\
\hline Wtrm1 & 18 & $1.30-1.55$ & 1.41 & 0.066 & 1 & 1.35 & - & - \\
\hline Wtlm1 & 16 & $1.40-1.65$ & 1.48 & 0.075 & - & - & - & - \\
\hline Hmdm1 & 14 & $3.20-4.00$ & 3.74 & 0.218 & 1 & 3.70 & - & - \\
\hline Hmdm3 & 19 & $3.60-4.40$ & 4.01 & 0.186 & 1 & 4.10 & - & - \\
\hline
\end{tabular}

morphologically almost identical to $M$. franconica from the early Miocene fauna from Petersbuch 28 and Petersbuch 62 (MN 3/4) of Germany (Rosina and Rummel 2012). Both the Megaderma franconica from Petersbuch 28 and the Megaderma from Petersbuch 2 and Erkertshofen 1 are only somewhat larger than the nominative form from WintershofWest (Tab. 2; see also Rosina and Rummel 2012: tab. S2, Ziegler 1993: 126, tab. 1). The morphological differences between these three taxa are not significant, thus we assign the studied fossil Megaderma from Petersbuch 2 and Erkertshofen 1 to $M$. franconica.
Family Rhinolophidae GrAY, 1825

Genus Rhinolophus LACÉPÈDE, 1799

Rhinolophus aff. lemanensis REVILLIOD, 1920

Text-fig. 2a-e

Materia 1. Erkertshofen 1: SNSB-BSPG 1962 XIX 4199, left mxl with M1-2; BSP 1962 XIX 4181, left mxl with P4-M2; BSP 1962 XIX 4182, left mxl with P4; BSP 1962 XIX 4179-4180, BSP 1962 XIX 4183-4191 (11 isolated upper teeth); BSP 1962 XIX 4176, right mnd with m3; BSP 
Table 2. Comparison of different early Miocene Megaderma species from Central Europe and the most important odontological features distinguishing the fossil species of the genus (in $\mathbf{m m}$, the size difference between maximum and minimum values, the arithmetic mean is in brackets).

\begin{tabular}{|c|c|c|c|c|c|c|c|c|c|c|}
\hline \multirow{2}{*}{ Species } & \multirow{2}{*}{ Locality, MN Zone } & \multicolumn{8}{|c|}{ Measurements } & \multirow{2}{*}{ References } \\
\hline & & LM1 & LM2 & LM3 & LC sup & WC sup & Lm1 & Lm3 & Wtlm3 & \\
\hline $\begin{array}{l}\text { Megaderma } \\
\text { franconica }\end{array}$ & $\begin{array}{c}\text { Petersbuch 2, } \\
\text { Germany, MN } 4\end{array}$ & \begin{tabular}{|c|}
$2.80-2.90$ \\
$(2.84)$ \\
\end{tabular} & \begin{tabular}{|c|}
$2.60-2.95$ \\
$(2.79)$
\end{tabular} & $\begin{array}{c}1.50-1.55 \\
(1.52)\end{array}$ & $\begin{array}{c}3.15-3.35 \\
(3.27)\end{array}$ & $\begin{array}{c}1.90-2.05 \\
(1.96)\end{array}$ & $\begin{array}{c}2.55-2.90 \\
(2.74)\end{array}$ & $\begin{array}{c}2.60-2.90 \\
(2.77)\end{array}$ & $\begin{array}{c}1.00-1.20 \\
(1.10)\end{array}$ & new data \\
\hline M. franconica & $\begin{array}{l}\text { Erkertshofen 1, } \\
\text { Germany, MN } 4\end{array}$ & - & 2.75 & - & - & - & $\approx 2.80$ & 2.80 & 1.00 & new data \\
\hline M. franconica & $\begin{array}{c}\text { Petersbuch 28, } \\
\text { Germany, MN } 3\end{array}$ & $\begin{array}{c}2.80-3.10 \\
(2.96)\end{array}$ & $\begin{array}{c}2.65-2.90 \\
(2.80)\end{array}$ & $\begin{array}{c}1.35-1.45 \\
(1.42)\end{array}$ & $\begin{array}{c}2.95-3.50 \\
(3.30)\end{array}$ & $\begin{array}{c}1.80-2.15 \\
(1.99)\end{array}$ & $\begin{array}{c}2.65-2.95 \\
(2.76)\end{array}$ & $\begin{array}{c}2.45-2.90 \\
(2.69)\end{array}$ & $\begin{array}{c}0.90-1.10 \\
(1.00)\end{array}$ & $\begin{array}{c}\text { Rosina and } \\
\text { Rummel } 2012\end{array}$ \\
\hline M. franconica & $\begin{array}{l}\text { Wintershof-West, } \\
\text { Germany, MN } 3\end{array}$ & \begin{tabular}{|c|}
$2.76-2.97$ \\
$(2.90)$
\end{tabular} & \begin{tabular}{|c|}
$2.87-3.00$ \\
$(2.93)$
\end{tabular} & 1.53 & $\begin{array}{c}3.04-3.46 \\
(3.17)\end{array}$ & $\begin{array}{c}1.85-2.31 \\
(2.02)\end{array}$ & $\begin{array}{c}2.73-2.99 \\
(2.82)\end{array}$ & $\begin{array}{c}2.80-3.03 \\
(2.93)\end{array}$ & - & Ziegler 1993 \\
\hline M. brailloni & $\begin{array}{c}\text { Bouzigues, } \\
\text { France, } \mathrm{MN} 2\end{array}$ & - & 3.26 & - & 3.35 & 2.3 & 2.9 & \begin{tabular}{|c|}
$2.95-3.00$ \\
$(2.98)$
\end{tabular} & - & Sigé 1968 \\
\hline M. lugdunensis & $\begin{array}{l}\text { Vieux Collonges, } \\
\text { France, MN } 5\end{array}$ & 2.60 & 2.60 & 1.00 & - & - & 2.60 & - & - & Sigé 1976 \\
\hline M. cf. franconica & $\begin{array}{c}\text { Stubersheim 3, } \\
\text { Germany, MN } 3\end{array}$ & - & - & - & - & - & 2.60 & 2.90 & 1.38 & Ziegler 1994 \\
\hline M. jaegeri & $\begin{array}{c}\text { Beni Mellal, Africa, } \\
\text { MN } 5 \text { or MN } 6\end{array}$ & $\begin{array}{c}2.05-2.10 \\
(2.07)\end{array}$ & - & - & 1.97 & 1.20 & \begin{tabular}{|c|}
$1.86-1.91$ \\
$(1.88)$
\end{tabular} & - & - & \multirow{3}{*}{ Sigé 1976} \\
\hline M. gaillardi & $\begin{array}{l}\text { Beni Mellal, Africa, } \\
\text { MN } 5 \text { or MN } 6\end{array}$ & - & 3.55 & 1.70 & 3.55 & 2.80 & - & 3.50 & 1.60 & \\
\hline M. gaillardi & $\begin{array}{c}\text { La Grive 7, } \\
\text { France, MN } 7\end{array}$ & - & - & - & - & - & 3.30 & 3.50 & - & \\
\hline
\end{tabular}

1962 XIX 4172-4175, 4177-4178 (6 isolated lower teeth).

Erkertshofen 2: BSP 1974 XIV 1167, left mxl with P4; BSP 1974XIV 1168, right mxl with P4; BSP 1974 XIV 1165, right mxl with M2-3; BSP 1974 XIV 1164, right mxl with M2-3; BSP 1974 XIV 1166, right mxl with M2; BSP 1974 XIV 1158-1163, 1169-1180, 1181-1187 (25 isolated upper teeth); BSP 1974 XIV 1139, left mnd with p4-m1; BSP 1974 XIV 1144, right mnd with p4; BSP 1974 XIV 1156-1157, right mnd with m3; BSP 1974 XIV 1137-1138, 1140-1141, 1143, 1147-1155 (14 isolated lower teeth).

Petersbuch 2: BSP 1976 XXII 5520, 5523, PCMRCh59-65, 108-109 (11 maxillary fragments with and without teeth); BSP 1976 XXII 5521, 5538, 5539, PCMRCh58a-v, 66, 67a-d, 68a-i, PCMRCh69a-i, 70a-j (58 isolated upper teeth); BSP 1976 XXII 5513-5517, 5531-5534, 5546, PCMRCh77a-e, 78a-h, 79, 80a-b (26 mandibles with teeth); BSP 1976 XXII 5525-5530, 55355537, 11071, PCMRCh80c-g, 81a-f, 82a-f, 83a-f, 84a-d, $85 \mathrm{a}-\mathrm{p}$ (53 isolated lower teeth).

\section{Me a surements. See Tab. 3.}

Description and comparison. The material is composed of fragments of the maxillary and mandibular bones with cheek teeth and isolated teeth. The fossils bear all the morphological traits typical of $R$. lemanensis as described in detail earlier (Rosina and Rummel 2012: 467468). Most of the fossil Rhinolophus species known from Europe exhibit significant differences in size. The dentition of the large Rhinolophus from Petersbuch 2, Erkertshofen 1 and Erkertshofen 2 is robust (Tabs 3,4). This species has the following odontological peculiarities: the talon of upper M1 protrudes slightly into the maxillary bone with a short tooth apophysis which is not a true root and has no alveolus. As a result, the alveolus of the lingual root of M1 has an additional posterolingual groove. The M2 also has a broad talon but it is smaller than that of the M1. Accordingly, the posterolingual groove of the lingual root of M2 is faint. In most specimens the p3 or its alveolus is reduced and displaced buccally (Text-fig. 2e). The degree of its displacement varies. This large species of Rhinolophus from Petersbuch 2, Erkertshofen 1 and Erkertshofen 2 compares well in morphology with the forms of $R$. aff. lemanensis from the early Miocene (MN 3) localities Wintershof-West, Stubersheim 3 (Ziegler 1993, 1994), Petersbuch 28 and Petersbuch 62 (Rosina and Rummel 2012). Rhinolophus lemanensis from the type locality Saint Gérand (MN 2a) is very sparsely represented (Revilliod 1920) and the size range of this species is unknown. However, the only known measurements of the $R$. lemanensis holotype and the two paratypes published by Revilliod (1920) and Ziegler (1993: 136) lie very close to the range of samples of $R$. aff. lemanensis from Petersbuch 2, Erkertshofen 1 and Erkertshofen 2 (compare with Tab. 3). Additionally, the nominative species $R$. lemanensis differs from all the above-discussed forms in having a less reduced $\mathrm{m} 3$. All forms of $R$. lemanensis s. str. overlap significantly in dimensions; there are no clear hiatuses. The nominative form of $R$. lemanensis from Saint Gérand has the smallest premolar, while the third molar is the largest of the later forms of $R$. aff. lemanensis from Wintershof-West and Stubersheim 3 and $R$. aff. lemanensis from Petersbuch 28 and Petersbuch 62. Thus, there is a gradual increase in size of the $\mathrm{p} 2$ but a reduction in $\mathrm{m} 3$ during the Neogene evolution of the rhinolophids (compare Tab. 3 with Ziegler 1993: 136). Since there is a significant overlap of the dimensions of all the discussed forms, as well as their significant morphological correspondence, we assign the samples from Petersbuch 2, Erkertshofen 1 and Erkertshofen 2 to R. lemanensis. The other forms of Rhinolophus, e.g. $R$. delphinensis, are slightly smaller than R. lemanensis s. str. (Ziegler 2003: 456, tab. 2). 


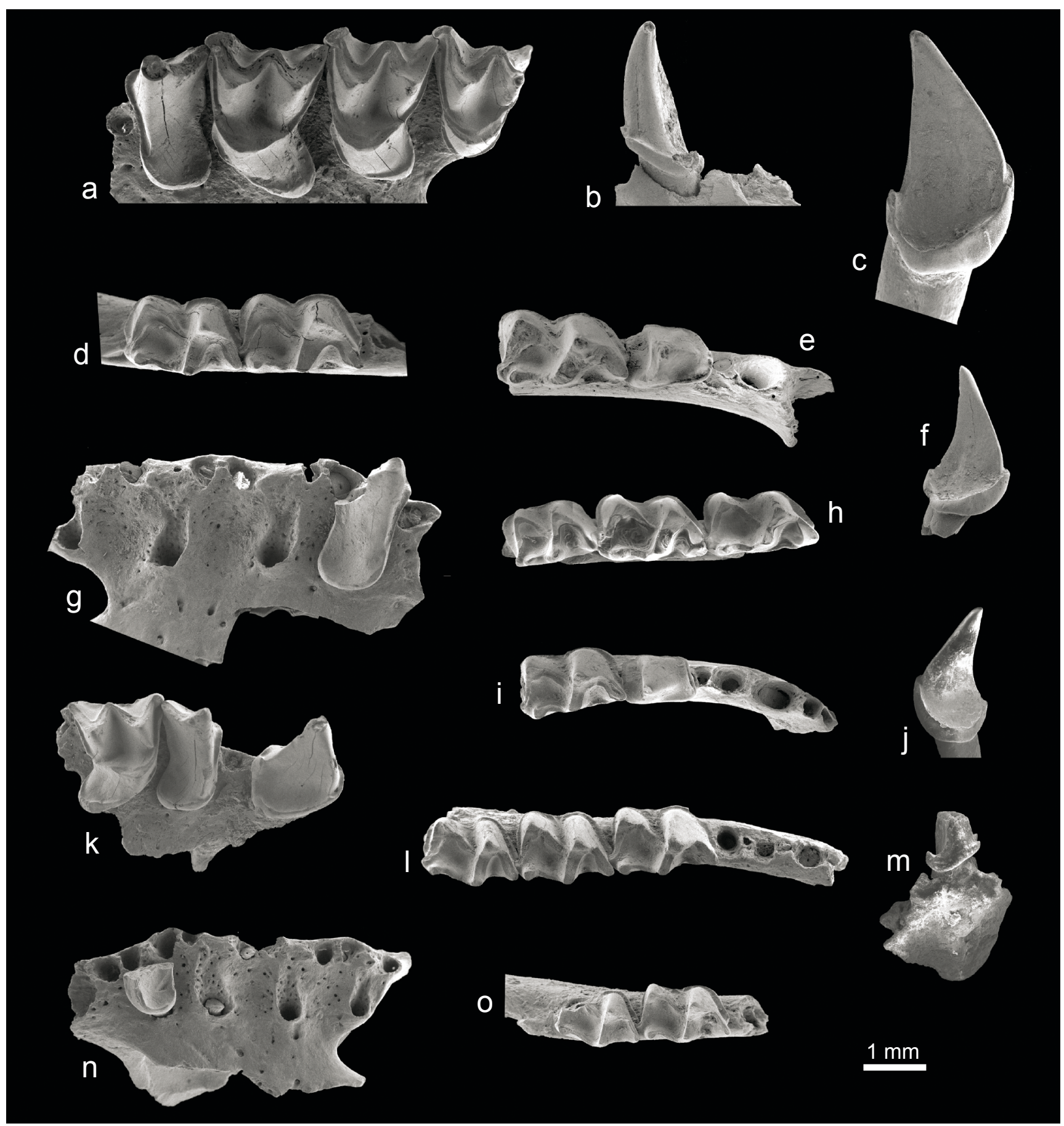

Text-fig. 2. a-e - Rhinolophus aff. lemanensis: a - PCMRCh65, left mxl with P4-M3, Petersbuch 2, ventral view; b - PCMRCh79 right mnd with $C$ inf., Petersbuch 2, lingual view; c - PCMRCh66, right C sup., Petersbuch 2, lingual view; d - BSP 1976 XXII 5515, left mnd with m2-3, Petersbuch 2, occlusal view; e - BSP 1974 XIV 1139, left mnd with p4-m1; Erkertshofen 2, occlusal view; f, i, k-m - R. dehmi: f - BSP 1974 XIV 1118, right C sup., Erkertshofen 2, lingual view; i - BSP 1976 XXII 5507, left mnd with p4-m1, Petersbuch 2, occlusal view; $\mathrm{k}$ - PCMRCh32, right mxl with $\mathrm{C}$ sup., P4-M1, Petersbuch 2, ventral view; l- PCMRCh37, left mnd with m1-2, Petersbuch 2, occlusal view; $m$ - PCMRCh89, right mnd with C inf., Petersbuch 2, lateral view; g-h - R. cf. delphinensis: g - BSP 1976 XXII 5522, right mxl with P4, Petersbuch 2, ventral view; h - BSP 1962 XIX 4153, left mnd with m1-3, Erkertshofen 1, occlusal view; j, n, o-R. grivensis: j - PCMRCh43, left C sup., Petersbuch 2, lingual view; n - BSP 1976 XXII 5543, left mxl, Petersbuch 2, ventral view; o - BSP 1976 XXII 5548, left mnd with m2-3, Petersbuch 2, occlusal view.

\section{Rhinolophus cf. delphinensis GAILLARD, 1899}

Text-fig. $2 \mathrm{~g}, \mathrm{~h}$

Mat e ria 1. Erkertshofen 1: BSP 1962 XIX 4150, left C inf.; BSP 1962 XIX 4152, left mnd with m1-2; BSP 1962 XIX 4153, left mnd with m1-3; BSP 1962 XIX 4155, left mnd with m3; BSP 1962 XIX 4156, right m1; BSP 1962
XIX 4158, left m2; BSP 1962 XIX 4160, left C sup.; BSP 1962 XIX 4166, left M1; BSP 1962 XIX 4167, left M1; BSP 1962 XIX 4171, left M2 sin.

Erkertshofen 2: BSP 1974 XIV 1099, right C inf.; BSP 1974 XIV 1142, left p4; BSP 1974 XIV 1145, right p4.

Petersbuch 2: BSP 1976 XXII 5522, right mxl with P4; BSP 1976 XXII 5524, right mxl with P4. 
Table 3. Rhinolophus aff. lemanensis from Petersbuch 2, Erkertshofen 1 and Erkertshofen 2, sample statistics of the teeth and jaws.

\begin{tabular}{|c|c|c|c|c|c|c|c|c|c|c|c|c|}
\hline \multirow{2}{*}{ Measur. } & \multicolumn{4}{|c|}{ Petersbuch 2} & \multicolumn{4}{|c|}{ Erkertshofen 1} & \multicolumn{4}{|c|}{ Erkertshofen 2} \\
\hline & $\mathrm{n}$ & $\mathbf{R}$ & $\mathbf{m}$ & $\mathbf{S}$ & $\mathrm{n}$ & $\mathbf{R}$ & $\mathbf{m}$ & $\mathbf{S}$ & $\mathbf{n}$ & $\mathbf{R}$ & $\mathbf{m}$ & $\mathbf{S}$ \\
\hline $\mathbf{L C}$ & 23 & $1.90-2.15$ & 2.05 & 0.059 & 2 & 2.00 & 2.00 & 0.000 & 6 & $2.00-2.15$ & 2.05 & 0.065 \\
\hline WC & 21 & $1.60-1.90$ & 1.74 & 0.086 & 2 & $1.65-1.70$ & 1.68 & 0.036 & 5 & $1.65-1.90$ & 1.76 & 0.096 \\
\hline $\mathrm{HC}$ & 15 & $3.60-4.10$ & 3.80 & 0.143 & 2 & $3.60-3.80$ & 3.70 & 0.141 & 3 & $3.50-4.15$ & 3.82 & 0.325 \\
\hline LP2 al. & 3 & $0.40-0.50$ & 0.47 & 0.058 & 2 & $0.40-0.45$ & 0.43 & 0.035 & 1 & 0.40 & - & - \\
\hline WP2 al. & 3 & $0.40-0.50$ & 0.45 & 0.050 & 2 & $0.40-0.45$ & 0.43 & 0.035 & 2 & $0.30-0.40$ & 0.35 & 0.071 \\
\hline LP4 & 9 & $1.40-1.65$ & 1.53 & 0.088 & 2 & $1.40-1.50$ & 1.45 & 0.071 & 1 & 1.40 & - & - \\
\hline WP4 & 7 & $2.00-2.15$ & 2.09 & 0.058 & 2 & $2.00-2.10$ & 2.05 & 0.071 & 2 & $1.80-2.10$ & 1.95 & 0.212 \\
\hline LP4-M3 & 1 & 6.35 & - & - & - & - & - & - & - & - & - & - \\
\hline LM1-M3 & 2 & $5.15-5.45$ & 5.30 & 0.212 & - & - & - & - & - & - & - & - \\
\hline LM1 & 15 & $2.00-2.15$ & 2.07 & 0.059 & 4 & $2.00-2.10$ & 2.03 & 0.05 & 3 & 2.00 & 2.00 & 0.000 \\
\hline WM1 & 16 & $2.25-2.65$ & 2.52 & 0.131 & 3 & $2.40-2.50$ & 2.43 & 0.058 & 4 & $2.40-2.65$ & 2.56 & 0.111 \\
\hline LM2 & 14 & $1.90-2.10$ & 1.97 & 0.073 & 1 & 1.90 & - & - & 6 & $1.75-2.05$ & 1.91 & 0.099 \\
\hline WM2 & 16 & $2.25-2.65$ & 2.40 & 0.106 & 1 & 2.40 & - & - & 6 & $2.20-2.55$ & 2.45 & 0.122 \\
\hline LM3 & 13 & $1.30-1.55$ & 1.44 & 0.073 & 5 & $1.45-1.60$ & 1.49 & 0.065 & 8 & $1.40-1.60$ & 1.46 & 0.079 \\
\hline WM3 & 10 & $2.10-2.30$ & 2.18 & 0.072 & 4 & $2.15-2.30$ & 2.20 & 0.071 & 7 & $2.00-2.25$ & 2.16 & 0.085 \\
\hline Lc & 17 & $1.10-1.30$ & 1.22 & 0.056 & 1 & 1.30 & - & - & 2 & $1.25-1.30$ & 1.28 & 0.035 \\
\hline Wc & 17 & $1.30-1.50$ & 1.38 & 0.059 & 1 & 1.40 & - & - & 2 & $1.40-1.50$ & 1.45 & 0.071 \\
\hline Hc & 12 & $2.20-2.70$ & 2.39 & 0.157 & 1 & 2.40 & - & - & 2 & 2.60 & 2.60 & 0.000 \\
\hline Lc-m1 & 2 & $5.65-6.00$ & 5.83 & 0.247 & - & - & - & - & - & - & - & - \\
\hline Lp2 & 4 & $1.15-1.25$ & 1.19 & 0.048 & - & - & - & - & 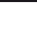 & - & - & - \\
\hline Wp2 & 4 & $1.05-1.15$ & 1.10 & 0.058 & - & - & - & - & - & - & - & - \\
\hline Lp4 & 8 & $1.35-1.55$ & 1.46 & 0.067 & 1 & 1.50 & - & - & 4 & $1.40-1.55$ & 1.49 & 0.075 \\
\hline Wp4 & 8 & $1.10-1.20$ & 1.15 & 0.038 & 1 & 1.25 & - & - & 5 & $1.10-1.25$ & 1.16 & 0.065 \\
\hline Lm1-2 & 3 & $4.20-4.35$ & 4.27 & 0.076 & - & - & - & - & - & - & - & - \\
\hline Lm2-3 & 3 & $3.90-3.95$ & 3.93 & 0.029 & - & - & - & - & - & - & - & - \\
\hline Lm3 & 18 & $1.70-2.10$ & 1.91 & 0.097 & 3 & $1.90-1.95$ & 1.93 & 0.029 & 4 & $1.85-2.00$ & 1.94 & 0.075 \\
\hline Wtrm3 & 19 & $1.10-1.30$ & 1.18 & 0.061 & 3 & $1.15-1.20$ & 1.18 & 0.029 & 4 & $1.10-1.25$ & 1.20 & 0.071 \\
\hline Wtlm3 & 19 & $0.95-1.20$ & 1.11 & 0.074 & 3 & $1.10-1.15$ & 1.13 & 0.029 & 4 & $1.10-1.15$ & 1.13 & 0.029 \\
\hline Lm2 & 18 & $2.00-2.25$ & 2.13 & 0.073 & - & - & - & - & 3 & 2.15 & 2.15 & 0.000 \\
\hline Wtrm2 & 19 & $1.15-1.35$ & 1.25 & 0.059 & - & - & - & - & 3 & $1.20-1.40$ & 1.30 & 0.100 \\
\hline Wtlm2 & 19 & $1.20-1.45$ & 1.31 & 0.072 & - & - & - & - & 3 & $1.30-1.40$ & 1.35 & 0.050 \\
\hline Lm1 & 18 & $2.00-2.35$ & 2.20 & 0.094 & 2 & $2.20-2.25$ & 2.23 & 0.035 & 5 & $2.15-2.25$ & 2.20 & 0.035 \\
\hline Wtrm1 & 18 & $1.05-1.40$ & 1.18 & 0.086 & 2 & 1.30 & 1.30 & 0.000 & 5 & $1.20-1.35$ & 1.28 & 0.067 \\
\hline WtIm1 & 18 & $1.10-1.45$ & 1.28 & 0.073 & 2 & 1.40 & 1.40 & 0.000 & 5 & $1.25-1.40$ & 1.34 & 0.065 \\
\hline Hmdm1 & 9 & $2.00-2.45$ & 2.29 & 0.139 & - & - & - & - & 1 & 2.10 & - & - \\
\hline Hmdm3 & 10 & $2.20-2.50$ & 2.39 & 0.097 & 1 & 2.50 & - & - & 1 & 2.45 & - & - \\
\hline
\end{tabular}

Me a surements. See Tab. 4.

Description and comparison. The preserved fossil jaw fragments bear all the morphological traits typical of Rhinolophus species and morphologically correspond to $R$. delphinensis already described in detail (see Ziegler 2003: 451-456). As was reported above many fossil Rhinolophus species known from Europe have significant differences in size (see also Ziegler 2003: 456, tab. 2). The middle-sized Rhinolophus from Petersbuch 2, Erkertshofen 1 and Erkertshofen 2 (Tab. 4) is significantly smaller than $R$. aff. lemanensis (Tab. 3) and larger than other small forms of Rhinolophus from Petersbuch 2, Erkertshofen 1 and Erkertshofen 2 (Tab. 4). The Rhinolophus from Petersbuch 2, Erkertshofen 1 and Erkertshofen 2 compares well in morphology with $R$. delphinensis from both the type locality La Grive 7 (see Text-fig. 2g-h; Gaillard 1899; for more detailed measurements see Mein 1964) and the middle Miocene sites of Petersbuch 6, 10, 18 and 31 (Ziegler 2003: fig. 1(1-6)). However, there are only a few fossils of this middle-sized Rhinolophus from Petersbuch 2, Erkertshofen 1 and Erkertshofen 2 and we assign them to $R$. cf. delphinensis. The other small-sized forms of Rhinolophus from Petersbuch 2, Erkertshofen 1 and Erkertshofen 2 are significantly smaller than the form of $R$. cf. delphinensis (see below; Tab. 4).

\section{Rhinolophus dehmi ZIEGLER, 1993}

Text-fig. 2f, i, k-m

Material. Erkertshofen 1: BSP 1962 XIX 4151, left C inf.; BSP 1962 XIX 4154, right mnd with p4; BSP 
Table 4. Small Rhinolophus species from Petersbuch 2 (P 2), Erkertshofen 1 (E 1) and Erkertshofen 2 (E 2), sample statistics of the teeth and jaws.

\begin{tabular}{|c|c|c|c|c|c|c|c|c|c|c|c|c|c|}
\hline \multirow{2}{*}{ Measur. } & \multirow{2}{*}{ Loc. } & \multicolumn{4}{|c|}{ R. grivensis } & \multicolumn{4}{|c|}{ R. dehmi } & \multicolumn{4}{|c|}{$R$. cf. delphinensis } \\
\hline & & $\mathbf{n}$ & $\mathbf{R}$ & $\mathbf{m}$ & $\mathbf{S}$ & $\mathbf{n}$ & $\mathbf{R}$ & $\mathbf{m}$ & $\mathbf{S}$ & $\mathbf{n}$ & $\mathbf{R}$ & $\mathbf{m}$ & $\mathbf{S}$ \\
\hline \multirow{3}{*}{ LC } & P 2 & 5 & $1.20-1.30$ & 1.26 & 0.042 & 46 & $1.40-1.80$ & 1.58 & 0.072 & - & - & - & - \\
\hline & E 1 & - & - & - & - & 3 & $1.55-1.75$ & 1.65 & 0.100 & 1 & 1.85 & - & - \\
\hline & E 2 & 1 & 1.30 & - & - & 7 & $1.40-1.80$ & 1.54 & 0.135 & - & - & - & - \\
\hline \multirow{3}{*}{ WC } & $\mathbf{P} 2$ & 5 & $0.90-1.05$ & 0.99 & 0.065 & 46 & $1.05-1.40$ & 1.19 & 0.058 & - & - & - & - \\
\hline & E 1 & - & - & - & - & 3 & $1.25-1.40$ & 1.33 & 0.076 & 1 & 1.40 & - & - \\
\hline & E 2 & 1 & 1.00 & - & - & 7 & $1.05-1.50$ & 1.20 & 0.147 & - & - & - & - \\
\hline \multirow{3}{*}{ HC } & $\mathbf{P} 2$ & 1 & 2.30 & - & - & 22 & $2.55-3.00$ & 2.80 & 0.131 & - & - & - & - \\
\hline & E 1 & - & - & - & - & 3 & $2.80-3.25$ & 3.07 & 0.236 & 1 & 3.15 & & \\
\hline & E 2 & - & - & - & - & 3 & $2.65-3.05$ & 2.87 & 0.202 & - & - & & \\
\hline \multirow{2}{*}{ LP4 } & $\mathbf{P} 2$ & 2 & 1.10 & - & - & 13 & $1.10-1.25$ & 1.18 & 0.049 & 2 & ca. $1.40-1.45$ & - & - \\
\hline & E 1 & - & - & - & - & 2 & $1.20-1.25$ & 1.23 & 0.035 & - & - & - & - \\
\hline \multirow{2}{*}{ WP4 } & $\mathbf{P} 2$ & 2 & 1.30 -ca. 1.35 & - & - & 14 & $1.45-1.70$ & 1.55 & 0.074 & 2 & ca. 2.00 & - & - \\
\hline & E 1 & - & - & - & - & 2 & $1.60-1.65$ & 1.63 & 0.035 & - & - & - & - \\
\hline LP4-M3 & $\mathbf{P} 2$ & - & - & - & - & 1 & ca. 4.95 & - & - & - & - & - & - \\
\hline LM1-M3 & P 2 & - & - & - & - & 1 & ca. 4.15 & - & - & - & - & - & - \\
\hline \multirow{3}{*}{ LM1 } & $\mathbf{P} 2$ & 1 & 1.45 & - & - & 20 & $1.55-1.70$ & 1.64 & 0.046 & - & - & - & - \\
\hline & E 1 & - & - & - & - & 1 & 1.70 & - & - & 2 & 1.85 & - & - \\
\hline & E 2 & - & - & - & - & 5 & $1.55-1.70$ & 1.61 & 0.055 & - & - & - & - \\
\hline \multirow{3}{*}{ WM1 } & $\mathbf{P 2}$ & 2 & $1.60-$ ca. 1.65 & - & - & 20 & $1.80-2.10$ & 1.95 & 0.092 & - & - & - & - \\
\hline & E 1 & - & - & - & - & 1 & 2.00 & - & - & 2 & $2.20-2.30$ & - & - \\
\hline & E 2 & - & - & - & - & 5 & $1.80-2.10$ & 1.93 & 0.110 & - & - & - & - \\
\hline \multirow{3}{*}{ LM2 } & $\mathbf{P} 2$ & 2 & 1.45 & - & - & 25 & $1.45-1.65$ & 1.54 & 0.047 & - & - & - & - \\
\hline & E 1 & 1 & 1.4 & - & - & - & - & - & - & 1 & 1.90 & - & - \\
\hline & E 2 & - & - & - & - & 7 & $1.50-1.60$ & 1.52 & 0.045 & - & - & - & - \\
\hline \multirow{3}{*}{ WM2 } & $\mathbf{P 2}$ & 2 & 1.65 & - & - & 26 & $1.70-1.95$ & 1.86 & 0.065 & - & - & - & - \\
\hline & E 1 & 1 & 1.60 & - & - & - & - & - & - & 1 & 2.15 & - & - \\
\hline & E 2 & - & - & - & - & 7 & ca. $1.75-1.85$ & 1.83 & 0.027 & - & - & - & - \\
\hline \multirow{2}{*}{ LM3 } & P 2 & 1 & 1.15 & - & - & 10 & $1.15-1.25$ & 1.17 & 0.036 & - & - & - & - \\
\hline & E 2 & - & - & - & - & 2 & 1.25 & - & - & - & - & - & - \\
\hline \multirow{2}{*}{ WM3 } & $\mathbf{P} 2$ & 1 & 145 & - & - & 9 & $1.65-1.75$ & 1.68 & 0.043 & - & - & - & - \\
\hline & E 2 & - & 1.45 & - & - & 1 & 1.65 & - & - & - & - & - & - \\
\hline & P 2 & - & - & - & - & 12 & $0.90-1.10$ & 0.99 & 0.061 & - & - & - & - \\
\hline Lc & E 1 & - & - & - & - & 3 & $0.95-1.15$ & 1.05 & 0.104 & 1 & 1.10 & - & - \\
\hline & E 2 & - & - & - & - & 5 & $0.95-1.20$ & 1.05 & 0.117 & 1 & 1.20 & - & - \\
\hline & $\mathbf{P 2}$ & - & - & - & - & 12 & $0.85-1.05$ & 0.95 & 0.062 & - & - & - & - \\
\hline Wc & E 1 & - & - & - & - & 3 & $1.10-1.15$ & 1.13 & 0.029 & 1 & 1.20 & - & - \\
\hline & E 2 & - & - & - & - & 5 & $0.90-1.20$ & 1.06 & 0.129 & - & - & - & - \\
\hline & $\mathbf{P} 2$ & - & - & - & - & 5 & $1.45-2.10$ & 1.84 & 0.251 & - & - & - & - \\
\hline Hc & E 1 & - & - & - & - & 1 & 2.20 & - & - & 1 & 2.25 & - & - \\
\hline & E 2 & - & - & - & - & 2 & $1.60-2.15$ & 1.88 & 0.389 & - & - & - & - \\
\hline & $\mathbf{P} 2$ & - & - & - & - & 10 & $1.05-1.30$ & 1.18 & 0.075 & - & - & - & - \\
\hline Lp4 & E 1 & - & - & - & - & 1 & 1.20 & - & - & - & - & - & - \\
\hline & E 2 & - & - & - & - & 1 & 1.15 & - & - & 2 & $1.25-1.35$ & 1.30 & 0.071 \\
\hline & $\mathbf{P} 2$ & - & - & - & - & 10 & $0.80-1.00$ & 0.90 & 0.058 & - & - & - & - \\
\hline Wp4 & E 1 & - & - & - & - & 1 & 0.95 & - & - & - & - & - & - \\
\hline & E 2 & - & - & - & - & 2 & $0.90-$ ca. 1.00 & 0.95 & 0.071 & 2 & 1.00 & - & - \\
\hline I $1-3$ & $\mathbf{P 2}$ & - & - & - & - & 2 & $4.45-4.65$ & 4.55 & 0.141 & - & - & - & - \\
\hline Lint- & E 1 & - & - & - & - & - & - & - & - & 1 & 5.25 & - & - \\
\hline Lm1-2 & $\mathbf{P} 2$ & - & - & - & - & 4 & $3.15-3.30$ & 3.24 & 0.075 & - & - & - & - \\
\hline & E 1 & - & - & - & - & - & - & - & - & 2 & $3.75-3.80$ & 3.78 & 0.030 \\
\hline
\end{tabular}


Table 4. continued.

\begin{tabular}{|c|c|c|c|c|c|c|c|c|c|c|c|c|c|}
\hline \multirow{2}{*}{ Measur. } & \multirow{2}{*}{ Loc. } & \multicolumn{4}{|c|}{ R. grivensis } & \multicolumn{4}{|c|}{ R. dehmi } & \multicolumn{4}{|c|}{$R$. cf. delphinensis } \\
\hline & & $\mathbf{n}$ & $\mathbf{R}$ & $\mathbf{m}$ & $\mathbf{S}$ & $\mathbf{n}$ & $\mathbf{R}$ & $\mathbf{m}$ & $\mathbf{S}$ & $\mathbf{n}$ & $\mathbf{R}$ & $\mathbf{m}$ & $\mathbf{S}$ \\
\hline \multirow{2}{*}{ Lm2-3 } & $\mathbf{P} 2$ & - & - & - & - & 5 & $2.80-3.20$ & 3.00 & 0.146 & - & - & - & - \\
\hline & E 1 & - & - & - & - & - & - & - & - & 1 & 3.40 & - & - \\
\hline \multirow{3}{*}{ Lm3 } & $\mathbf{P} 2$ & 1 & 1.45 & - & - & 23 & $1.40-1.65$ & 1.50 & 0.063 & - & - & - & - \\
\hline & E 1 & - & - & - & - & - & - & - & - & 2 & 1.70 & - & - \\
\hline & E 2 & - & - & - & - & 2 & $1.40-1.45$ & 1.43 & 0.035 & - & - & - & - \\
\hline \multirow{3}{*}{ Wtrm3 } & $\mathbf{P} 2$ & 1 & 0.85 & - & - & 22 & $0.85-0.95$ & 0.91 & 0.041 & - & - & - & - \\
\hline & E 1 & - & - & - & - & - & - & - & - & 2 & $1.00-1.10$ & 1.05 & 0.071 \\
\hline & E 2 & - & - & - & - & 2 & 0.95 & - & - & - & - & - & - \\
\hline \multirow{3}{*}{ Wtlm3 } & P2 & 1 & 0.80 & - & - & 22 & $0.80-1.05$ & 0.89 & 0.062 & - & - & - & - \\
\hline & E 1 & - & - & - & - & - & - & - & - & 2 & $1.00-1.10$ & 1.05 & 0.071 \\
\hline & E 2 & - & - & - & - & 2 & 0.90 & - & - & - & - & - & - \\
\hline \multirow{3}{*}{ Lm2 } & $\mathbf{P} 2$ & 2 & 1.55 & - & - & 22 & $1.55-1.75$ & 1.65 & 0.054 & - & - & - & - \\
\hline & E 1 & - & - & - & - & 1 & 1.6 & - & - & 3 & $1.85-1.90$ & 1.88 & 0.029 \\
\hline & E 2 & - & - & - & - & 3 & $1.65-1.75$ & 1.68 & 0.058 & - & - & - & - \\
\hline \multirow{3}{*}{ Wtrm2 } & $\mathbf{P} 2$ & 2 & 0.85 & - & - & 23 & $0.90-1.10$ & 0.95 & 0.051 & - & - & - & - \\
\hline & E 1 & - & - & - & - & 1 & 0.95 & - & - & 3 & $1.00-1.20$ & 1.10 & 0.100 \\
\hline & E 2 & & - & - & - & 3 & $0.90-1.00$ & 0.95 & 0.050 & - & - & - & - \\
\hline \multirow{3}{*}{ Wtlm2 } & $\mathbf{P} 2$ & 2 & 0.95 & - & - & 24 & $1.00-1.15$ & 1.06 & 0.040 & - & - & - & - \\
\hline & E 1 & - & - & - & - & 1 & 1.10 & - & - & 3 & $1.15-1.25$ & 1.18 & 0.058 \\
\hline & E 2 & - & - & - & - & 3 & $1.05-1.10$ & 1.08 & 0.029 & - & - & - & - \\
\hline \multirow{3}{*}{ Lm1 } & $\mathbf{P 2}$ & 1 & 1.60 & - & - & 24 & $1.65-1.85$ & 1.76 & 0.067 & - & - & - & - \\
\hline & E 1 & - & - & - & - & 1 & 1.70 & - & - & 3 & $1.95-2.00$ & 1.98 & 0.029 \\
\hline & E 2 & & - & - & - & 4 & $1.75-1.90$ & 1.81 & 0.063 & - & - & - & - \\
\hline \multirow{3}{*}{ Wtrm1 } & $\mathbf{P 2}$ & 1 & 0.85 & - & - & 24 & $0.85-1.00$ & 0.93 & 0.044 & - & - & - & - \\
\hline & E 1 & - & - & - & - & 1 & 0.90 & - & - & 3 & $1.00-1.15$ & 1.07 & 0.076 \\
\hline & E 2 & - & - & - & - & 5 & $0.95-1.00$ & 0.96 & 0.022 & - & - & - & - \\
\hline \multirow{3}{*}{ Wtlm1 } & $\mathbf{P 2}$ & - & 0.90 & - & - & 24 & $0.95-1.10$ & 1.02 & 0.042 & - & - & - & - \\
\hline & E 1 & - & - & - & - & 1 & 1.00 & - & - & 3 & $1.10-1.20$ & 1.13 & 0.058 \\
\hline & E 2 & - & - & - & - & 5 & $1.00-1.10$ & 1.04 & 0.042 & - & - & - & - \\
\hline \multirow{2}{*}{ Hmdm1 } & P 2 & - & - & - & - & 13 & $1.60-2.10$ & 1.83 & 0.160 & - & - & - & - \\
\hline & E 1 & - & - & - & - & 1 & 1.75 & - & - & 1 & 1.80 & - & - \\
\hline \multirow{2}{*}{ Hmdm3 } & $\mathbf{P 2}$ & - & - & - & - & 7 & $1.60-2.20$ & 1.79 & 0.213 & - & - & - & - \\
\hline & E 1 & - & - & - & - & - & - & - & - & 2 & ca. $1.80-2.00$ & 1.90 & 0.141 \\
\hline
\end{tabular}

1962 XIX 4157, right m1; BSP 1962 XIX 4159, left m2; BSP 1962 XIX 4150, left C inf.; BSP 1962 XIX 4151, left C inf.; BSP 1962 XIX 4161, right C sup.; BSP 1962 XIX 4162, left C sup.; BSP 1962 XIX 4163, left C sup.; BSP 1962 XIX 4164, right P4; BSP 1962 XIX 4165, right P4; BSP 1962 XIX 4168, left M1; BSP 1962 XIX 4169, left M1 (damaged); BSP 1962 XIX 4170, right M1 (damaged).

Erkertshofen 2: BSP 1974 XIV 1109, 1097, 1098, 11001108, 1110-1112, 1146, 1188 (16 isolated lower teeth); BSP 1974 XIV 1113, right mnd with m3; BSP 1974 XIV 11221124, 1126-1136, 1115-1121 (21 isolated upper teeth); BSP 1974 XIV 1125, right mxl with M1.

Petersbuch 2: BSP 1976 XXII 5504-5507, 5518, 5547, 5549-5552, PCMRCh37-39, 51b,

54a-i, 57e, 86f, 89 (26 mandibles with teeth); BSP 1976 XXII 11067a-f, 11068a-m, 11070, PCMRCh24-26, PCMRCh50a-g, 51a, 52a-d, 53, 55a-e, 56a-f, 57a-b, 57d, $57 \mathrm{f}-\mathrm{h}, 86 \mathrm{a}-\mathrm{e}$ (58 isolated lower teeth); BSP 1976 XXII 5519, 5541, 5542, PCMRCh32-34, 46a-c (9 maxillary fragments with teeth); BSP 1976 XXII 5544a-i, 5545a-d, PCMRCh28, 73, 35-36, 40a-r, 41a-w, 44a-1, 45a-q, 46d-1, 47a-g, 71-76 (105 isolated upper teeth).

\section{Me a surements. See Tab. 4.}

Des c ription. The shape of the fossil jaw fragments are typical for the Rhinolophus. The preserved remains morphologically correspond to $R$. dehmi described in detail earlier (see Rosina and Rummel 2012: 468, Ziegler 1993: 136-140). The upper canine is semilunar in occlusal view with a flat lingual surface and a well-developed cingulum (Text-fig. 2k). The P4 has a marked talon which protrudes posterolingually. The M1 differs from M2 by having a shorter preparacrista and a more developed talon on the posterolingual side of the crown. The lower canine is crescentshaped in occlusal view and surrounded by a well-developed cingulum which forms a small anterolingual broadening and a distinct distolingual cuspule (Text-fig. 2m). According to the alveoli, the $\mathrm{p} 2$ was large with a single root and the 
p3 was displaced buccally from the midline of the toothrow (Text-fig. 2i, 1). The $\mathrm{m} 3$ talonid is only slightly smaller than the trigonid (Text-fig. 21). This Rhinolophus species from Petersbuch 2, Erkertshofen 1 and Erkertshofen 2 is considerably smaller than both the $R$. cf. delphinensis and $R$. aff. lemanensis from the same sites (Tabs 3, 4). At the same time, it is significantly larger than $R$. grivensis (Tab. 4), but compares well in morphology with $R$. dehmi from the type locality Wintershof-West (Ziegler 1993) and was related to this species.

\section{Rhinolophus grivensis DEPÉRET, 1892 \\ Text-fig. $2 \mathrm{j}, \mathrm{n}-\mathrm{O}$} M2.

Mat e ria 1. Erkertshofen 1: BSP 1962 XIX 4194, left

Erkertshofen 2: BSP 1974 XIV 1114, left C sup.

Petersbuch 2: BSP 1976 XXII 5543, left mxl with P4; PCMRCh55a, rightm1; PCMRCh56a, leftm2; PCMRCh56b, right $\mathrm{m} 2$; $\mathrm{PCMRCh} 57 \mathrm{c}$, right $\mathrm{m} 3$; $\mathrm{PCMRCh} 48$, right $\mathrm{M} 3$; PCMRCh49a, left M1 (damaged); PCMRCh49b, left M1; PCMRCh49c, left M2; PCMRCh49d, right M2; PCMRCh27, left P4; PCMRCh29, right $\mathrm{C}$ sup.; PCMRCh30, right $\mathrm{C}$ sup.; PCMRCh31, left $\mathrm{C}$ sup.; PCMRCh42, right $\mathrm{C}$ sup.; PCMRCh43, left C sup.

Me a surements. See Tab. 4.

Description and comparison. The preserved fossils share all the morphological traits of Rhinolophus species: the upper canine is semilunar in occlusal view with a well-developed cingulum (Text-fig. 2j), p3 was displaced buccally from the midline of the tooth-row, and the $\mathrm{m} 3$ talonid is only slightly smaller than the trigonid (Text-fig. 2o). However, there is a significant space between the canine and P4 in the maxillary (Text-fig. 2n). This is the smallest and most gracile Rhinolophus species from the studied localities (Tab. 4) and morphologically corresponds to $R$. grivensis (e.g. Ziegler 2003: 456-459).

\section{Superfamily Vespertilionoidea GraY, 1821 Family Vespertilionidae GraY, 1821}

\section{Genus Hanakia HoRÁČEK, 2001}

\section{Hanakia agadjaniani Rosina et RuMmel, 2012} Figs $3 \mathrm{a}-\mathrm{g}, \mathrm{i}, 1,4$

M a teri a 1. Erkertshofen 2: BSP 1974 XIV 1194, left C sup.; BSP 1974 XIV 1195, right C sup.; BSP 1974 XIV 1192, right mxl with P4-M3; BSP 1974 XIV 1193, right mxl with P4-M2; BSP 1974 XIV 1197, right P4; BSP 1974 XIV 1198, right M1; BSP 1974 XIV 1196, right C sup.; BSP 1974 XIV 993, left mnd with talonid m2 and m3; BSP 1974 XIV 994, right mnd with m3; BSP 1974 XIV 995, right mnd with m2-3; BSP 1974 XIV 1189, right mnd with m2; BSP 1974 XIV 1191, left m1; BSP 1974 XIV 1190, left C inf.; BSP 1974 XIV 1111, right $\mathrm{m} 1$.

Petersbuch 2: BSP 1976 XXII 5512a-q, PCMRCh93a-j, PCMRCh94a-h, PCMRCh95a-i, PCMRCh96a-f, PCMRCh97a-f, PCMRCh98a-h, PCMRCh102a-c, PCMRCh114, PCMRCh8, PCMRCh9 (66 isolated upper teeth); BSP 1976 XXII 5488-5489, BSP 1976 XXII 5493-5497, PCMRCh90-92 (10 maxillary fragments with teeth); BSP 1976 XXII 5490-5492, PCMRCh10-11, BSP 1976 XXII 5498-5503, BSP 1976 XXII 5509h, BSP 1976 XXII 5511d-f, PCMRCh103a-i, PCMRCh104a-c (23 mandibles with teeth); BSP 1976 XXII 5508a-o, BSP 1976 XXII 5509a-g, BSP 1976 XXII 5510a-c, BSP 1976 XXII 5511a-c, PCMRCh99, PCMRCh100a-d, PCMRCh101, PCMRCh104d-f, PCMRCh105a-h, PCMRCh106a-g, PCMRCh107a-u (73 isolated lower teeth).

Me a surements. See Tab. 5.

Description and comparison. These specimens from Erkertshofen 2 and Petersbuch 2 show all the typical features of the genus Hanakia, particularly the robust construction of the cranium while the dental formula corresponds to Myotis. However, in its dentition the talonid of $\mathrm{m} 3$ is considerably reduced and the incisive row is markedly compressed. The mandible is robust and the preserved alveoli of i1-3 suggest that the incisors were similar in size, positioned very close to each other and that the i3 was buccally displaced (e.g. BSP 1976 XXII 5490; Text-fig. 4b). The ascending ramus is nearly at right angles to the body of the mandible and the masseteric fossa is very deep (e.g. BSP 1976 XXII 5492; Text-fig. 4d). The lower canine has a notable mesolingual cingular cuspid and is surrounded by a well-developed cingulum (e.g. PCMRCh10; Text-fig. 4c). The crown of the p2 has two small anterior and posterior tubercles. The $\mathrm{p} 4$ is quadrangular in occlusal view, surrounded by a distinct cingulum and has small anterior and posterior tubercles on the lingual side of the crown (e.g. BSP 1976 XXII 5491; Text-fig. 4e). All lower molars are myotodont with distinctly curved paralophids. The talonid of the $\mathrm{m} 3$ is markedly reduced (e.g. PCMRCh11; Text-fig. 4f). Hanakia from Erkertshofen 2 and Petersbuch 2 differs from H. fejfari from Merkur-North (= Ahníkov, MN 3, Czech Republic; Horáček 2001) in being smaller (Tab. 6) and in having larger p2-3 (compare with Horáček 2001: 139, figs 18, 19). The morphology of Hanakia from Erkertshofen 2 and Petersbuch 2 is almost identical to that of $H$. cf. agadjaniani ("Myotis cf. antiquus"; for details see Rosina and Rummel 2012) from Wintershof-West (Ziegler 1993) and from Stubersheim 3 (Ziegler 1994). However, the fossils under study differ from $H$. cf. agadjaniani in having a less reduced premolar tooth-row but a more robust mandibular body, in having a more reduced $\mathrm{m} 3$ talonid and M3, and in a lack of the additional conules and lophs on the upper molars (Text-figs 3, 4). Hanakia from Erkertshofen 2 and Petersbuch 2 differs from the H. antiquus (La Grive 7, MN 7, France, Gaillard 1899) in having a less reduced premolar tooth-row and a more robust mandibular body (Hmdm1 of M. antiquus is only $1.5 \mathrm{~mm}$; compare with Tab. 5). Moreover, the $\mathrm{m} 3$ talonid of $H$. antiquus is only slightly reduced in size (Gaillard 1899, Viret 1951: 18, fig. 3) also distinguishing this species from the specimens under study. Its M1-2 have paraand metalophs (Baudelot 1972: 50, tab. 2) and the M3 is less reduced (Viret 1951: 18, fig. 2) in contrast to the fossils from Erkertshofen 2 and Petersbuch 2. Morphologically these fossils from Erkertshofen 2 and Petersbuch 2 correspond to the type specimens of $H$. agadjaniani from Petersbuch 62 (Text-figs 3, 4; Rosina and Rummel 2012: 468-470). There are remains of another form of Hanakia in the localities of Petersbuch 2, Erkertshofen 1 and Erkertshofen 2, which 


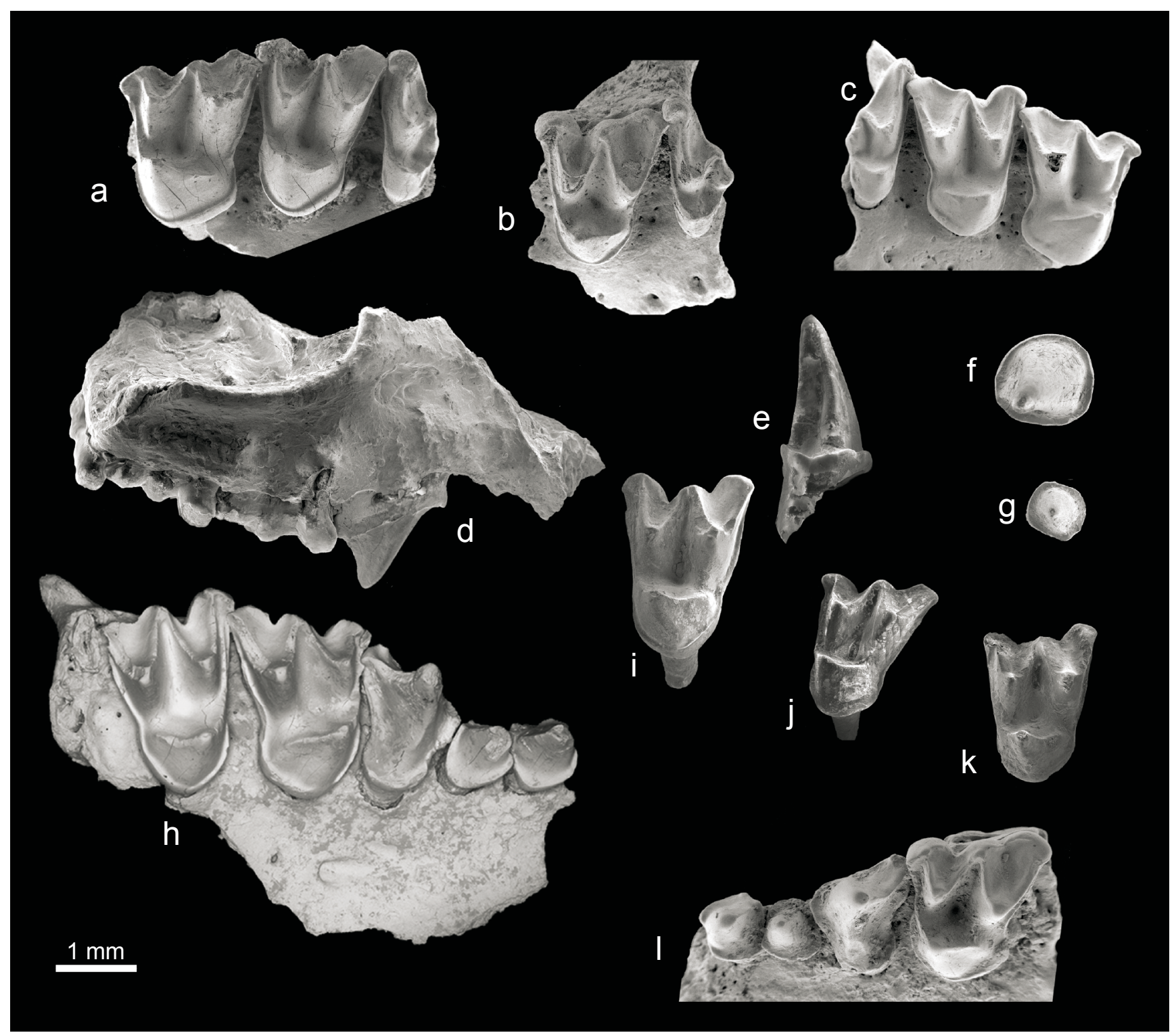

Text-fig. 3. Upper jaw fragments. a-g, i, l - Hanakia agadjaniani: a - PCMRCh90, left mxl with M1-3, Petersbuch 2, ventral view; b - PCMRCh91, left mxl with M2-3, Petersbuch 2, ventral view; c - BSP 1976 XXII 5488, right mxl with M1-3, Petersbuch 2, ventral view; d - BSP 1974 XIV 1192, right mxl with P4-M3, Erkertshofen 2, ventral view; e - PCMRCh9, right C sup., Petersbuch 2, lingual view; f - PCMRCh8, right $\mathrm{C}$ sup., Petersbuch 2, occlusal view; g - PCMRCh99, left P2, Petersbuch 2, occlusal view; i - PCMRCh114, left M2, Petersbuch 2, occlusal view; I - BSP 1976 XXII 5489, left mxl with P2-M2, Petersbuch 2, ventral view; h-H. agadjaniani, NMA P62/0334, right mxl with P2-M2, Petersbuch 62, ventral view; j, $\mathrm{k}-H$. aff. antiquus, Petersbuch 2: j - PCMRCh2, left M1, occlusal view; $k$ - PCMRCh1, left M2, occlusal view.

are noticeably smaller in size than $H$. agadjaniani from Petersbuch 2 and Erkertshofen 2 and differs in some other morphological features (see below).

\section{Hanakia aff. antiquus (GAILLARD, 1899)} Text-fig. $3 \mathrm{j}-\mathrm{k}$

Material and measurements. Erkertshofen 1: BSP 1962 XIX 4196, left M2, $1.60 \times 2.10$

Petersbuch 2: PCMRCh1, left M2, $\approx 1.50 \times \approx 2.00$; PCMRCh2, left M1, $1.60 \times 1.95 ;$ PCMRCh3, left M1, $1.40 \times 1.70$.

Erkertshofen 2: BSP 1974 XIV 1196, right C sup., $1.05 \times 1.10 \times 1.90$

Description and comparison. These fossils from Petersbuch 2, Erkertshofen 1 and Erkertshofen 2
(Text-fig. 3) morphologically correspond to $H$. agadjaniani, but are noticeably smaller in size (Tab. 6). Moreover, the trigon basin of the upper molar (specimen PCMRCh2) from Petersbuch 2 is closed (Text-fig. 3J), similar to Eptesicus aurelianensis from Stubersheim 3 (Ziegler 1994: 113, fig. 6), which is significantly smaller in size (compare with Ziegler 1994: 112). This specimen from Petersbuch 2 morphologically is closest to $H$. aff. antiquus (Tab. 6; compare with specimen Mer91C43, Horáček 2001: 133, fig. 14). Another fossil upper molar (specimen PCMRCh1) differs from $E$. aurelianensis in being larger and in having an open trigon basin without metaloph (Text-fig. 3K; compare with Ziegler 1994: 113, fig. 6). The studied upper molars differ from those of the species of $H$. antiquus from $\mathrm{La}$ Grive 7 in lacking the para- and metalophs (compare with Baudelot 1972: 50, tab. 2). 


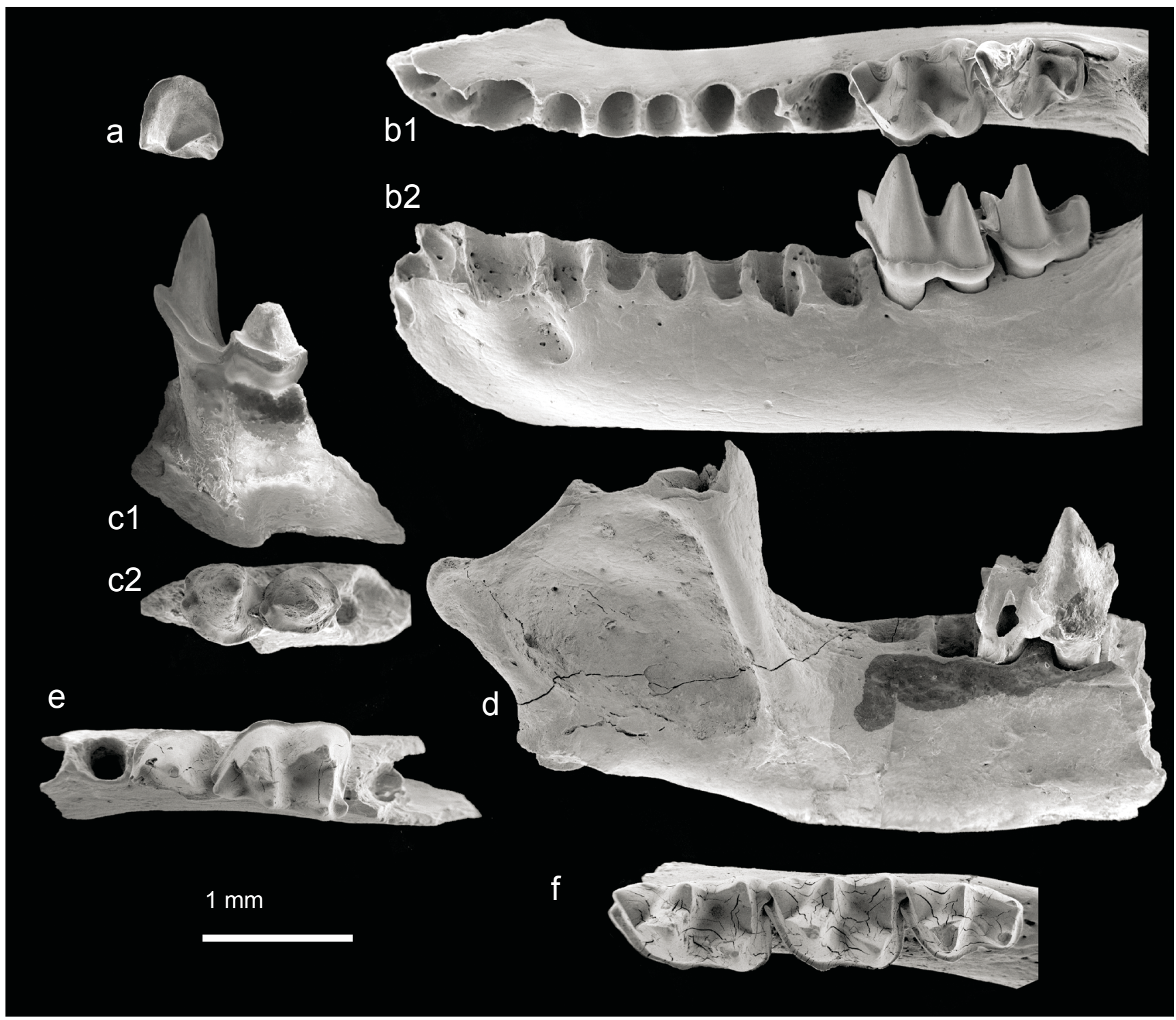

Text-fig. 4. Lower jaw fragments. Hanakia agadjaniani: a - PCMRCh101, left p2, Petersbuch 2, occlusal view; b - BSP 1976 XXII 5490, left mnd with m2-3, Petersbuch 2, occlusal (b1) and lateral (b2) views; c- PCMRCh10, right mnd with C inf.-p2, Petersbuch 2, lingual (c1) and occlusal (c2) views; d - BSP 1976 XXII 5492, right mnd with m2, Petersbuch 2, lateral view; e - BSP 1976 XXII 5491, right mnd with p4-m1; Petersbuch 2, occlusal view; f - PCMRCh11, left mnd with m1-3, Petersbuch 2, occlusal view.

\section{Genus Plecotus É. Geoffroy SaINT-HiLaIRe, 1813}

\section{Plecotus ef. atavus TopáL, 1987}

Text-figs $5 \mathrm{a}, 6 \mathrm{c}$

Material and measurements. Erkertshofen 1 : SNSB-BSPG 1962 XIX 4201, right p4, $0.75 \times 0.65$.

Petersbuch 2: PCMRCh3, left M1, $1.40 \times 1.70$.

Description and comparison. The molar (specimen PCMRCh3) from Petersbuch 2 is characterised by a closed trigon basin due to the postprotocrista which gradually merges with the postcingulum while the metaloph is absent (Text-fig. 5a). Paraconule, paraloph and hypocone are absent. These are typical signs of the upper molars of plecotin bats (Rosina et al. 2019). The studied M1 differs from the M1 of the late Miocene Barbastella maxima Rosina, Kruskop et Semenov, 2019 from Gritsev (MN 9, Ukraine; Rosina et al. 2019) in lacking the meta- and paralophs (Textfig. 5a, f). The cingulum from the distal side of the protocone of this upper molar from Petersbuch 2 is well developed and forms some hypoconal undulation (Text-fig. 5a), which makes this fossil form similar to the modern representatives of the modern American genus Corynorphinus (Text-fig. 5e). In morphological details, however, the specimen PCMRCh3 is most similar to the upper molars of the representatives of the group P. atavus s. str. from the Miocene localities of Europe (Text-fig. 5b-d).

The crown of the p4 (specimen SNSB-BSPG 1962 XIX 4201) from Erkertshofen 1 is shortened, the lingual cingulum is poorly developed and there is an only slightly pronounced anterolingual process of the cingulum. Its anterior root is slightly reduced compared with the posterior. These characters differentiate the p4 from Erkertshofen 1 from Myotis or Submyotodon (compare with Ziegler 2003: 478, fig. 6(1-2)). It is also distinguished from Miostrellus petersbuchensis Rosina et RuMmel, 2012 in having a more rounded crown in cross-section, and less developed anterolingual cuspid, as well as in having a reduced first 
Table 5. Hanakia agadjaniani from Petersbuch 2 and Erkertshofen 1, sample statistics of the teeth and jaws.

\begin{tabular}{|c|c|c|c|c|c|c|c|c|}
\hline \multirow{2}{*}{ Measur. } & \multicolumn{4}{|c|}{ Petersbuch 2} & \multicolumn{4}{|c|}{ Erkertshofen 1} \\
\hline & $\mathbf{n}$ & $\mathbf{R}$ & $\mathbf{m}$ & $S$ & $\mathbf{n}$ & $\mathbf{R}$ & $\mathbf{m}$ & $S$ \\
\hline LC & 16 & $1.20-1.40$ & 1.31 & 0.064 & 2 & 1.30 & 1.30 & - \\
\hline WC & 16 & $1.15-1.25$ & 1.20 & 0.036 & 2 & $1.15-1.20$ & 1.18 & 0.035 \\
\hline HC & 8 & $2.05-2.25$ & 2.17 & 0.075 & 2 & $2.00-2.45$ & 2.23 & 0.318 \\
\hline LP2 & 4 & $0.85-1.10$ & 0.95 & 0.093 & - & - & - & - \\
\hline WP2 & 4 & $0.85-0.95$ & 0.90 & 0.035 & - & - & - & - \\
\hline LP2 al. & 1 & 0.55 & - & - & 1 & 0.60 & - & - \\
\hline WP2 al. & 1 & 0.60 & - & - & 1 & 0.60 & - & - \\
\hline LP3 & 2 & $0.75-0.90$ & 0.83 & 0.075 & - & - & - & - \\
\hline WP3 & 2 & $0.75-0.80$ & 0.78 & 0.025 & - & - & - & - \\
\hline LP3 al. & 1 & 0.65 & - & - & 1 & 0.55 & - & - \\
\hline WP3 al. & 1 & 0.50 & - & - & 1 & 0.55 & - & - \\
\hline LP4 & 8 & $1.35-1.55$ & 1.43 & 0.061 & 3 & $1.35-1.50$ & 1.42 & 0.080 \\
\hline WP4 & 8 & $1.25-1.35$ & 1.32 & 0.043 & 3 & $1.25-1.35$ & 1.30 & 0.050 \\
\hline LP4-M3 & - & - & - & - & 1 & 5.10 & - & - \\
\hline LM1-M3 & 3 & $3.95-4.30$ & 4.15 & 0.147 & 1 & 4.00 & - & - \\
\hline LM1 & 20 & $1.70-1.85$ & 1.78 & 0.054 & 2 & $1.70-1.75$ & 1.73 & 0.040 \\
\hline WM1 & 21 & $1.90-2.20$ & 2.06 & 0.073 & 2 & $1.90-1.95$ & 1.93 & 0.040 \\
\hline LM2 & 19 & $1.65-1.90$ & 1.73 & 0.057 & 2 & $1.40-1.60$ & 1.50 & 0.141 \\
\hline WM2 & 21 & $2.10-2.45$ & 2.25 & 0.093 & 2 & $2.05-2.15$ & 2.10 & 0.071 \\
\hline LM3 & 9 & $0.75-0.95$ & 0.83 & 0.058 & 1 & 0.80 & - & - \\
\hline WM3 & 8 & $1.90-2.15$ & 1.98 & 0.083 & 1 & 1.90 & - & - \\
\hline Lc & 20 & $1.00-1.15$ & 1.08 & 0.046 & 1 & 1.10 & - & - \\
\hline We & 22 & $1.00-1.20$ & 1.13 & 0.054 & 1 & 1.10 & - & - \\
\hline Hc & 18 & $1.60-1.90$ & 1.76 & 0.072 & 1 & 1.75 & - & - \\
\hline Lp2 & 6 & $0.85-1.10$ & 1.02 & 0.051 & - & - & - & - \\
\hline Wp2 & 5 & $0.80-1.95$ & 0.89 & 0.054 & - & - & - & - \\
\hline Lp4 & 7 & $1.15-1.30$ & 1.26 & 0.052 & - & - & - & - \\
\hline Wp4 & 7 & $0.90-0.95$ & 0.92 & 0.025 & - & - & - & - \\
\hline Lm1-2 & 3 & $3.40-3.55$ & 3.47 & 0.062 & - & - & - & - \\
\hline Lm2-3 & 7 & $2.90-2.15$ & 3.02 & 0.096 & 1 & 3.10 & - & - \\
\hline Lm1-3 & 2 & $4.65-4.75$ & 4.70 & 0.050 & - & - & - & - \\
\hline Lm3 & 15 & $1.30-1.55$ & 1.42 & 0.057 & 3 & $1.30-1.45$ & 1.35 & 0.087 \\
\hline Wtrm3 & 14 & $0.85-0.95$ & 0.92 & 0.036 & 3 & $0.85-0.95$ & 0.90 & 0.050 \\
\hline Wtlm3 & 16 & $0.50-0.75$ & 0.62 & 0.063 & 3 & $0.50-0.60$ & 0.55 & 0.050 \\
\hline Lm2 & 30 & $1.65-1.90$ & 1.76 & 0.058 & 2 & $1.75-1.85$ & 1.80 & 0.071 \\
\hline Wtrm2 & 30 & $0.90-1.15$ & 1.04 & 0.076 & 2 & $1.00-1.05$ & 1.03 & 0.035 \\
\hline Wtlm2 & 29 & $1.00-1.20$ & 1.11 & 0.060 & 3 & $1.10-1.15$ & 1.12 & 0.029 \\
\hline Lm1 & 30 & $1.65-1.90$ & 1.78 & 0.051 & 1 & 1.65 & - & - \\
\hline Wtrm1 & 30 & $0.85-1.15$ & 0.97 & 0.070 & 2 & 0.90 & 0.90 & - \\
\hline Wtlm1 & 32 & $1.00-1.25$ & 1.14 & 0.049 & 1 & 1.05 & - & - \\
\hline Hmdm1 & 12 & $2.15-2.50$ & 2.30 & 0.101 & - & - & - & - \\
\hline Hmdm3 & 13 & $2.25-2.70$ & 2.45 & 0.134 & 3 & $2.10-2.60$ & 2.53 & 0.250 \\
\hline
\end{tabular}

root (compare with Rosina and Rummel 2012: 471, fig. 5E, F). It differs from the p2 of Miniopterus rummelii by a less developed anterolingual cuspid (Ziegler 2003: 486, fig. $7(1-2)$ ), and in having a shorter protolophid than the buccal cristid (Text-fig. 6c). In precisely these characteristics the $\mathrm{p} 4$ from Erkertshofen 1 is most similar to the $\mathrm{p} 4$ of $P$. atavus (Text-fig. 6) as well as in size (compare with Rosina et al. 2019: 3, tab. 1). However, the species affiliation of these single fossil teeth from Erkertshofen 1 and Petersbuch 2 could not be confirmed with complete confidence.

\section{Genus Myotis KAuP, 1829}

\section{Myotis cf. reductus ZIEGLER 2003 \\ Text-fig. 7a-e, h, i}

Material and measurements. Erkertshofen 1: SNSB-BSPG 1962 XIX 4200, left mnd without teeth: C inf. (al.) $0.65 \times 0.45 ; \mathrm{p} 2$ (al.) $0.50 \times 0.40 ; \mathrm{p} 3$ (al.) $0.35 \times 0.30$; Hmdm1 1.50 .

Erkertshofen 2: BSP 1974 XIV 1208, right M1, $1.40 \times$ 1.55; BSP 1974 XIV 1209, left M2, $21.2 \times \approx 1.75$; BSP 1974 
Table 6. Comparison of Hanakia s. str. from Petersbuch 2, Erkertshofen 1 and Erkertshofen 2 with some different fossil forms of Hanakia species (in mm, the size difference between maximum and minimum values, the arithmetic mean is in brackets).

\begin{tabular}{|c|c|c|c|c|c|c|c|c|c|c|}
\hline \multirow{2}{*}{ Species } & \multirow{2}{*}{$\begin{array}{l}\text { Locality, } \\
\text { MN Zone }\end{array}$} & \multicolumn{8}{|c|}{ Measurements } & \multirow{2}{*}{ References } \\
\hline & & LC sup. & LM1 & LM2 & LM3 & LC inf. & Lm2-3 & Lm1 & Lm3 & \\
\hline \multirow{2}{*}{ H. agadjaniani } & $\begin{array}{c}\text { Erkertshofen } 2 \\
\text { MN 4b }\end{array}$ & 1.30 & $\begin{array}{c}1.70-1.75 \\
(1.73)\end{array}$ & $\begin{array}{c}1.40-1.60 \\
(1.50)\end{array}$ & 0.80 & 1.00 & 3.10 & 1.65 & $\begin{array}{c}1.30-1.45 \\
(1.35)\end{array}$ & \multirow{5}{*}{ New data } \\
\hline & $\begin{array}{c}\text { Petersbuch 2, } \\
\text { MN 4a }\end{array}$ & \begin{tabular}{|c|}
$1.20-1.40$ \\
$(1.31)$
\end{tabular} & $\begin{array}{c}1.70-1.85 \\
(1.78) \\
\end{array}$ & \begin{tabular}{|c|}
$1.65-1.90$ \\
$(1.73)$
\end{tabular} & \begin{tabular}{|c|}
$0.75-0.95$ \\
$(0.83)$ \\
\end{tabular} & \begin{tabular}{|c|}
$1.00-1.15$ \\
$(1.08)$
\end{tabular} & \begin{tabular}{|c|}
$2.90-3.15$ \\
$(3.02)$ \\
\end{tabular} & $\begin{array}{c}1.65-1.90 \\
(1.78) \\
\end{array}$ & \begin{tabular}{|c|}
$1.30-1.55$ \\
$(1.42)$
\end{tabular} & \\
\hline \multirow{3}{*}{ H. aff. antiquus } & $\begin{array}{c}\text { Erkertshofen } 2, \\
\text { MN 4b }\end{array}$ & 1.05 & - & - & - & - & - & - & - & \\
\hline & $\begin{array}{c}\text { Erkertshofen 1, } \\
\text { MN 4b }\end{array}$ & - & - & 1.60 & - & - & - & - & - & \\
\hline & $\begin{array}{l}\text { Petersbuch 2, } \\
\text { MN 4a }\end{array}$ & - & 1.40 & $\begin{array}{c}\begin{array}{c}1.50-1.60 \\
(1.55)\end{array} \\
\end{array}$ & - & - & - & - & - & \\
\hline H. agadjaniani & $\begin{array}{c}\text { Petersbuch } 62 \\
\text { MN 3/4 }\end{array}$ & $\begin{array}{c}1.20-1.40 \\
(1.31)\end{array}$ & $\begin{array}{c}1.60-1.95 \\
(1.80)\end{array}$ & $\begin{array}{c}1.60-1.85 \\
(1.73)\end{array}$ & $\begin{array}{c}0.80-0.95 \\
(0.88)\end{array}$ & $\begin{array}{c}1.10-1.20 \\
(1.14)\end{array}$ & $\begin{array}{c}2.90-3.30 \\
(3.08)\end{array}$ & $\begin{array}{c}1.55-1.90 \\
(1.76)\end{array}$ & $\begin{array}{c}1.30-1.55 \\
(1.44)\end{array}$ & $\begin{array}{c}\text { Rosina and } \\
\text { Rummel } 2012\end{array}$ \\
\hline H. cf. agadjaniani & $\begin{array}{l}\text { Wintershof-West, } \\
\text { MN } 3\end{array}$ & $\begin{array}{c}1.02-1.09 \\
(1.06)\end{array}$ & 1.83 & 1.72 & 0.85 & $\begin{array}{c}1.24-1.40 \\
(1.31)\end{array}$ & - & $\begin{array}{c}1.7-1.80 \\
(1.76)\end{array}$ & $\begin{array}{c}1.35-1.48 \\
(1.41)\end{array}$ & Ziegler 1993 \\
\hline H. cf. agadjaniani & $\begin{array}{c}\text { Stubersheim 3, } \\
\text { MN } 3\end{array}$ & \begin{tabular}{|c|}
$1.12-1.47$ \\
$(1.29)$
\end{tabular} & $\begin{array}{c}1.60-1.96 \\
(1.76)\end{array}$ & \begin{tabular}{|c|}
$1.55-1.78$ \\
$(1.66)$
\end{tabular} & $\begin{array}{c}0.74-0.90 \\
(0.82)\end{array}$ & \begin{tabular}{|c|}
$1.02-1.47$ \\
$(1.28)$
\end{tabular} & - & $\begin{array}{c}1.57-1.85 \\
(1.75)\end{array}$ & \begin{tabular}{|c|}
$1.33-1.64$ \\
$(1.45)$
\end{tabular} & Ziegler 1994 \\
\hline H. antiquus & $\begin{array}{c}\text { La Grive } 7, \\
\text { MN } 7\end{array}$ & - & 1.80 & - & - & - & - & 1.80 & - & $\begin{array}{l}\text { Gaillard } 1899, \\
\text { Baudelot } 1972\end{array}$ \\
\hline H. cf. antiquus & \multirow{2}{*}{$\begin{array}{l}\text { Merkur-North, } \\
\text { MN } 3\end{array}$} & - & - & - & - & 1.10 & $\begin{array}{c}2.50-5.00 \\
(3.27)\end{array}$ & $\begin{array}{c}1.52-1.73 \\
(1.63)\end{array}$ & $\begin{array}{c}1.30-1.48 \\
(1.38)\end{array}$ & \multirow{2}{*}{ Horáček 2001} \\
\hline $\begin{array}{l}\text { Hanakia fejfari } \\
\text { holotype }\end{array}$ & & - & - & - & - & - & 3.80 & - & 1.75 & \\
\hline
\end{tabular}

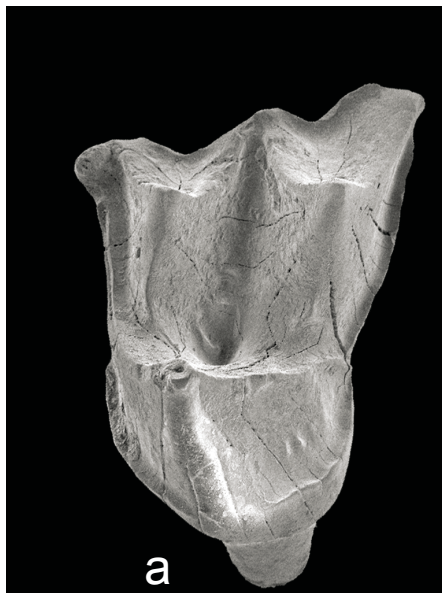

a

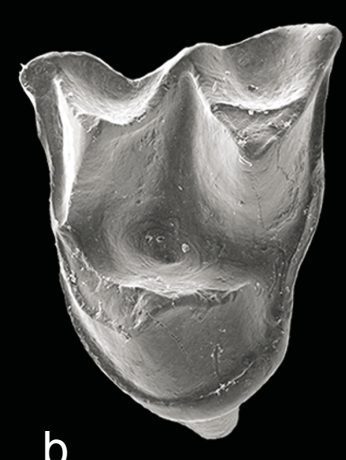

b

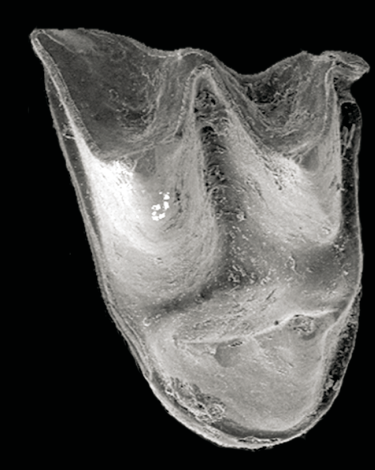

C

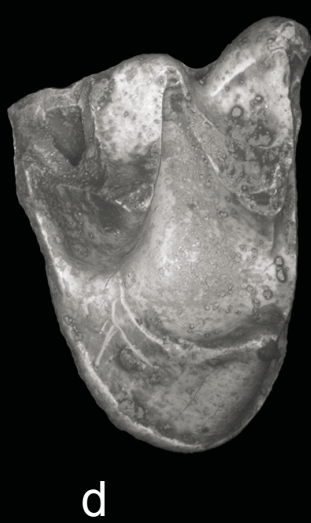

d

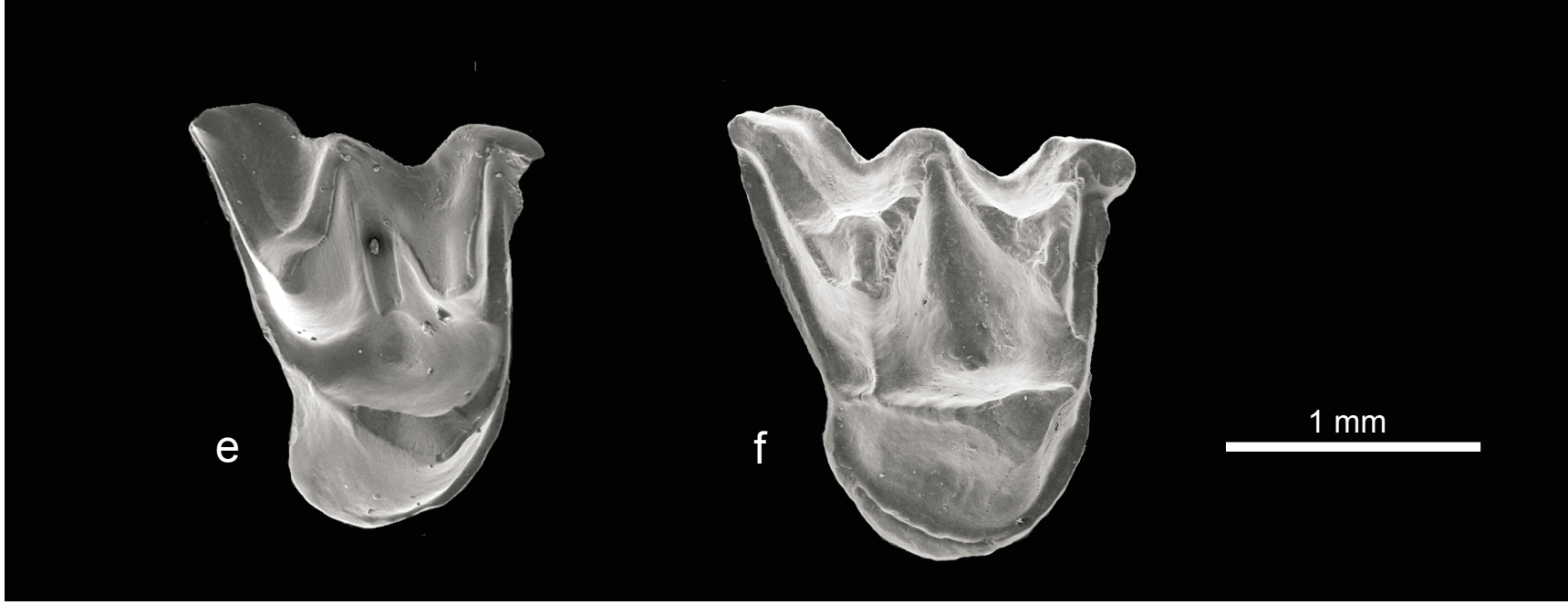

Text-fig. 5. Upper molars of different fossil and recent Plecotini, occlusal view. a - Plecotus cf. atavus, PCMRCh3, left M1, Petersbuch 2; b - P. aff. atavus, Ch/G-175, left M1, Gritsev; c - P. auritus, ZMMU S-174773, right M1, recent; d - P. schoepfelii, NMA P62/0114, right M2, Petersbuch 62; e - Corynorhinus townsendii, ZMMU S-105677, right M1, recent; f - Barbastella maxima, Ch/G-001, right M1, Gritsev. 


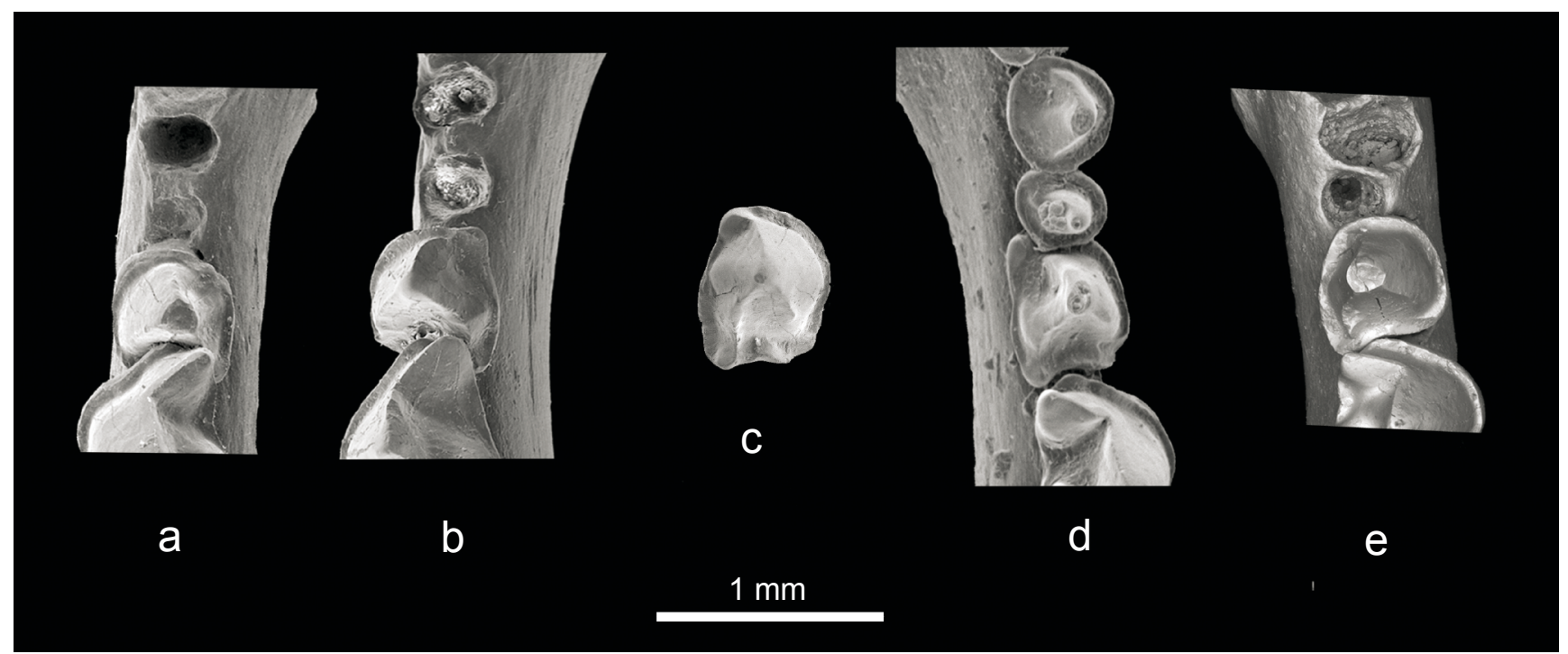

Text-fig. 6. Lower jaw fragments of different fossil and recent Plecotini, occlusal view. a, b -Plecotus aff. atavus, fragment of left mnd with p4, Gritsev: a - specimen Ch/G-096; b - specimen Ch/G-104; c - P. cf. atavus, SNSB-BSPG 1962 XIX 4201, right p4, Erkertshofen 1, occlusal view; d - P. auritus, ZMMU S-174773, fragment of right mnd with p4, recent; e - P. schoepfelii, NMA P28/0478, fragment of right mnd with p4, Petersbuch 28.

XIV 1202, left p4, $0.80 \times 0.75 ;$ BSP 1974 XIV 1201, right $\mathrm{C}$ inf., $0.85 \times 0.80 \times 1.20$; BSP 1974 XIV 1199 , left C inf., $0.85 \times 0.80 \times 1.20$.

Petersbuch 2: BSP 1976 XXII 4799, right mnd with p4-m3: p2 (al.) $0.45 \times 0.45 ;$ p3 (al.) $0.45 \times 0.45 ; \mathrm{p} 4$ $0.85 \times 0.70 ; \mathrm{m} 11.35 \times 0.78 \times 0.80 ; \mathrm{m} 21.30 \times 0.85 \times 0.85$; m3 $1.28 \times 0.80 \times 0.45 ; \mathrm{Hmdm} 1$ 1.43; PCMRCh87, right m3, $1.20 \times 0.70 \times 0.65$.

Description and comparison. The crowns of the upper molars (specimens BSP 1974 XIV 1208 and BSP 1974 XIV 1209; Text-fig. 7a, b) show the paraconules and the distinct para- and metalophs. The postprotocrista extends to the lingual bases of the metacones and forms a small hypocone. Thus, the trigon basins of the molars are closed. All these are the typical features of Myotis. Both upper molars from Erkertshofen 2 correlate well with each other in size, implying their belonging to one taxon. They differ from the early Miocene $M$. cf. sanctialbani from Petersbuch 28 (MN 3, Germany; Rosina and Rummel 2012: tab. S1, supplementary data) in being smaller in size and in having a small hypocone (Text-fig. 7a-c, Tab. 7; Rosina and Rummel 2012: 474, fig. 6). They are smaller in size than the middle Miocene M. bavaricus ZIEGLER, 2003 (MN 7/8, Petersbuch 6, 10, 18; Ziegler 2003; Tab. 7). The early Miocene $M$. aff. murinoides (e.g. SMNS 45742.1) from Stubersheim 3 (MN 3, Germany; Ziegler 1994: 104) and from Oberdorf 3 (MN 4, Austria; Ziegler 1998) is smaller than the fossils under study, which also show a more developed paraconule and paraloph in the upper molar crowns (compare Text-fig. 7A-C with Ziegler 1994: 107, pl. 3, figs 1-3, Ziegler 1998: 95, pl. 8, figs 11, 12). In comparison with the fossils from Erkertshofen 2, the species M. murinoides (LARTET, 1851) from Sansan (MN 6, France) is smaller in size (Baudelot 1972: 24, tab.) and has a more developed hypocone (Baudelot 1972: 35, fig. 14). Thus, the upper molars from Erkertshofen 2 are most similar to those of M. aff. reductus from Petersbuch 28 (MN 3; Text-fig. 7a-c).
The edentulous mandibular fragment (specimen SNSBBSPG 1962 XIX 4200) from Erkertshofen 1 has three alveoli of the lower incisors while the alveolus of the third incisor is the largest (Text-fig. 7e). The alveolus of the canine is roundish. The small premolars are single-rooted. The roundish alveoli of the small premolars are all located at the midline of the tooth-row and their form indicates that the crown of $\mathrm{p} 3$ was smaller than $\mathrm{p} 2$. The root alveoli of the p4 are about the same size that indicates an elongated crown in this tooth (Text-fig. 7E). All these are typical features of the genus of Myotis. The alveoli of the small premolars of another mandibular fragment (specimen BSP 1976 XXII 4799) from Petersbuch 2 are similar in size, the paralophids of the myotodontal lower molars are less curved and the talonid of $\mathrm{m} 3$ is less reduced. All these are features indicate its affiliation to Myotis. It differs from Submyotodon in being larger, in having larger alveoli of the $\mathrm{i} 3$ and p2, and also in having a more elongated crown on the p4 (Ziegler 2003: 478, fig. 6). Both mandibular fragments from Erkertshofen 1 and Petersbuch 2 are smaller than $M$. bavaricus and differ from it in having smaller p2-3 (compare with the holotype of $M$. bavaricus p2 (al.) $0.60 \times 0.65$ ). Thus, the mentioned mandibular fragments are closest in size to $M$. aff. reductus from Petersbuch 28 and Petersbuch 62 (Tab. 7; Rosina and Rummel 2012).

As typical for Myotis, the crowns of the lower canines from Erkertshofen 2 (specimens BSP 1974 XIV 1201 and BSP 1974 XIV 1199) are uncompressed in anteroposterior direction. They have pronounced, but low, anterolingual cuspules of cingulid, not strongly pressed to the tooth bodies, and a flattening of the distal part of the cingulids (Text-fig. 7d). Morphologically they are similar to the lower canines of $M$. bavaricus but somewhat smaller in size (Tab. 7). For morphological and also biostratigraphic reasons, the lower canines from Erkertshofen 2 were attributed to $M$. aff. reductus from Petersbuch 28, which is somewhat smaller than $M$. bavaricus in size, but the lower canines of which are unknown. 


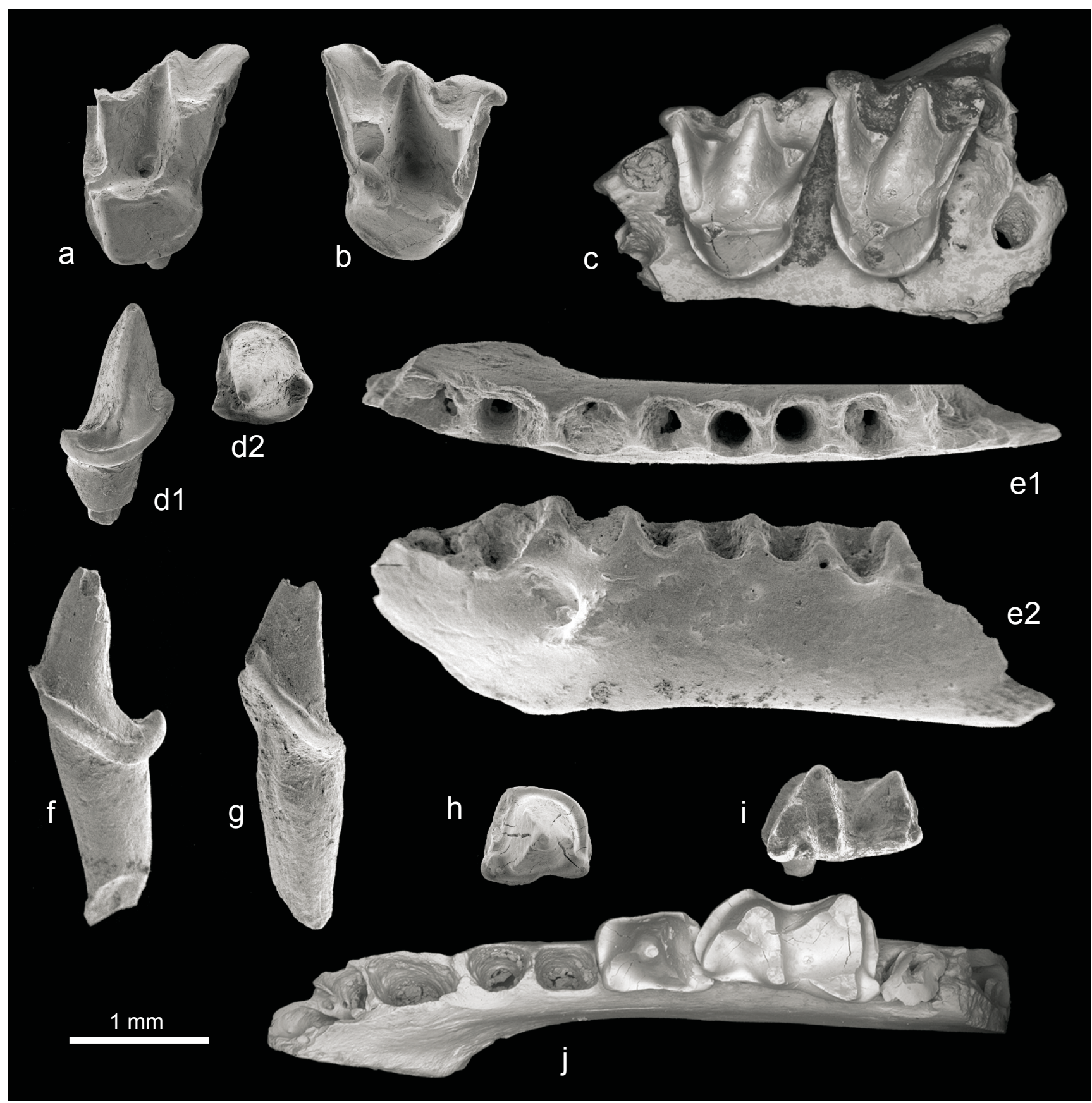

Text-fig. 7. a-e, h, i - Myotis cf. reductus: a - BSP 1974 XIV 1209, left M2, Erkertshofen 2, occlusal view; b - BSP 1974 XIV 1208 , right M1, Erkertshofen 2, occlusal view; c - left maxillary fragment with M1-2, NMA P28/0345, Petersbuch 28, ventral view; d - BSP 1974 XIV 1199, left C inf., Erkertshofen 2, lingual (c1) and occlusal (c2) views; e - SNSB-BSPG 1962 XIX 4200 , left mnd without teeth; Erkertshofen 1, occlusal (e1) and lateral (e2) views; h - BSP 1974 XIV 1202, left p4, Erkertshofen 2, occlusal view; i - PCMRCh87, right m3, Petersbuch 2, occlusal view; f, g- cf. Myotis sp., right C inf., Petersbuch 2, lingual view: f - PCMRCh25, g - PCMRCh88; j - M. aff. reductus, NMA P62/0331, right dentary fragment with p4-m1, Petersbuch 62, occlusal view.

The p4 from Erkertshofen 2 (specimen BSP 1974 XIV 1202) has an elongated crown with a well-developed cingulid (Text-fig. 7h) that is typical of many Myotis species. It is somewhat smaller in size than the p4 of both the $M$. bavaricus and $M$. aff. murinoides (specimen NHMW 1997z0024/0001/2; Ziegler 1998: 95, pl. 8, fig. 10) from Oberdorf 3. On the other hand, the p4 from Erkertshofen 2 is larger than M. murinoides from Sansan (Tab. 7). Morphologically it is closest to $M$. aff. reductus from Petersbuch 28 and Petersbuch 62, especially in the twolobed shape of the crown with pronounced anterolingual and posterolingual cuspules (Text-fig. 7h; Rosina and Rummel 2012: 474, fig. 6E). However, the poor preservation of the fossils, only allows tentatively proposing their taxonomic unity with $M$. aff. reductus.

\section{cf. Myotis sp. \\ Text-fig. 7f, g}

Material and measurements. Petersbuch 2: PCMRCh25, right $\mathrm{C}$ inf., $\approx 0.85 \times 0.95$; $\mathrm{PCMRCh88}$, right C inf., $1.05 \times 0.95$. 
Description and comparison. The crowns of the lower canines from Petersbuch 2 are uncompressed in the anteroposterior direction with a pronounced, but low, anterolingual cuspid of the cingulid, not strongly pressed to the tooth bodies (Text-fig. 7f, g), which suggests their assignment to Myotis. They are very similar to the lower canines of $M$. cf. reductus from Erkertshofen 2 but somewhat larger in size (Tab. 7).

\section{Genus Eptesicus RAFInesQue, 1820}

\section{Eptesicus cf. aurelianensis ZIEGLER, 1993 Text-fig. $8 \mathrm{~g}$}

Material and measurements. Erkertshofen 1: BSP 1962 XIX 4197, right M2, $1.45 \times 1.90$.

Petersbuch 2: BSP 1980 XXII 5366, right M2, $\approx 1.45 \times$ $\approx 1.90$

Description and comparison. The M2 crowns from Erkertshofen 1 and Petersbuch 2 show a welldeveloped cingulum and paralophs (Text-fig. 8g1). The absence of a large hypocone suggests that the tooth belongs to a vespertilionid bat. Both molars have para- and metalophs but lack the paraconules. The trigon basins are closed, the hypocones are weakly developed (Text-fig. 8g). The upper molars from Erkertshofen 1 and Petersbuch 2 share these features with Miostrellus or Eptesicus. Nevertheless, they are larger than all Miostrellus species (Tab. 8) and, thus, more similar in size to E. aurelianensis (compare with e.g. specimen SMNS 45744 H1; Ziegler 1994: 113, pl. 5, fig. 6). However, they differ from E. aurelianensis in having some undulated metaloph and a less developed hypocone (Textfig. $8 \mathrm{~g} 2$ ).

\section{Genus Miostrellus RACHL, 1983}

\section{Miostrellus cf. noctuloides (LARTET, 1851) Text-fig. 8b-f, k, n}

Material and measurements. Erkertshofen 1: BSP 1962 XIX 4195, left M1, $1.30 \times 1.55$; BSP 1962 XIX 4198, right M3, $0.85 \times 1.55$; BSP 1962 XIX 4192, right mnd with m1-2: Lm1-2 2.70, m2 $1.40 \times 0.80 \times 0.90, \mathrm{~m} 1$ $1.45 \times 0.80 \times 0.90, \mathrm{Hmdm} 1$ 1.70, Hmdm3 1.60; BSP 1962 XIX 4193, right m2, $1.40 \times 0.85 \times 0.95$.

Erkertshofen 2: BSP 1974 XIV 1204, left C sup., $1.00 \times$ $0.90 \times 1.65$; BSP 1974 XIV 1200, right $\mathrm{C}$ inf., $0.65 \times$ $0.75 \times 1.30$.

Petersbuch 2: PCMRCh4, left C sup., $1.05 \times 0.90 \times 1.70$; PCMRCh23, right P4, $1.10 \times 1.15$; BSP 1977 XXII 4800, right mnd with $\mathrm{m} 2-3: \mathrm{m} 21.30 \times 0.75 \times 0.75, \mathrm{~m} 31.13 \times$ $0.73 \times 0.55, \mathrm{Hmdm} 1 \approx 1.75$; BSP 1976 XXII 5361 , left M1, $1.35 \times 1.45$; BSP 1977 XXII 5362, right M1, $1.30 \times 1.40$; BSP 1977 XXII 5363, right M1, $\approx 1.25 \times 1.40$; BSP 1978 XXII 5364, right M1, $1.25 \times 1.35$.

Description and comparison. The upper molars from Erkertshofen 1 have a well-developed cingulum and paraloph. The crown of the M1 (specimen BSP 1962 XIX 4195; Text-fig. 8c) show a weak metaloph, thus, the trigon basin is closed. Both upper molars from Erkertshofen 1 are significantly larger than those of $M$. risgoviensis and smaller than those of Eptesicus aurelianensis (Tab. 8). They differ from 


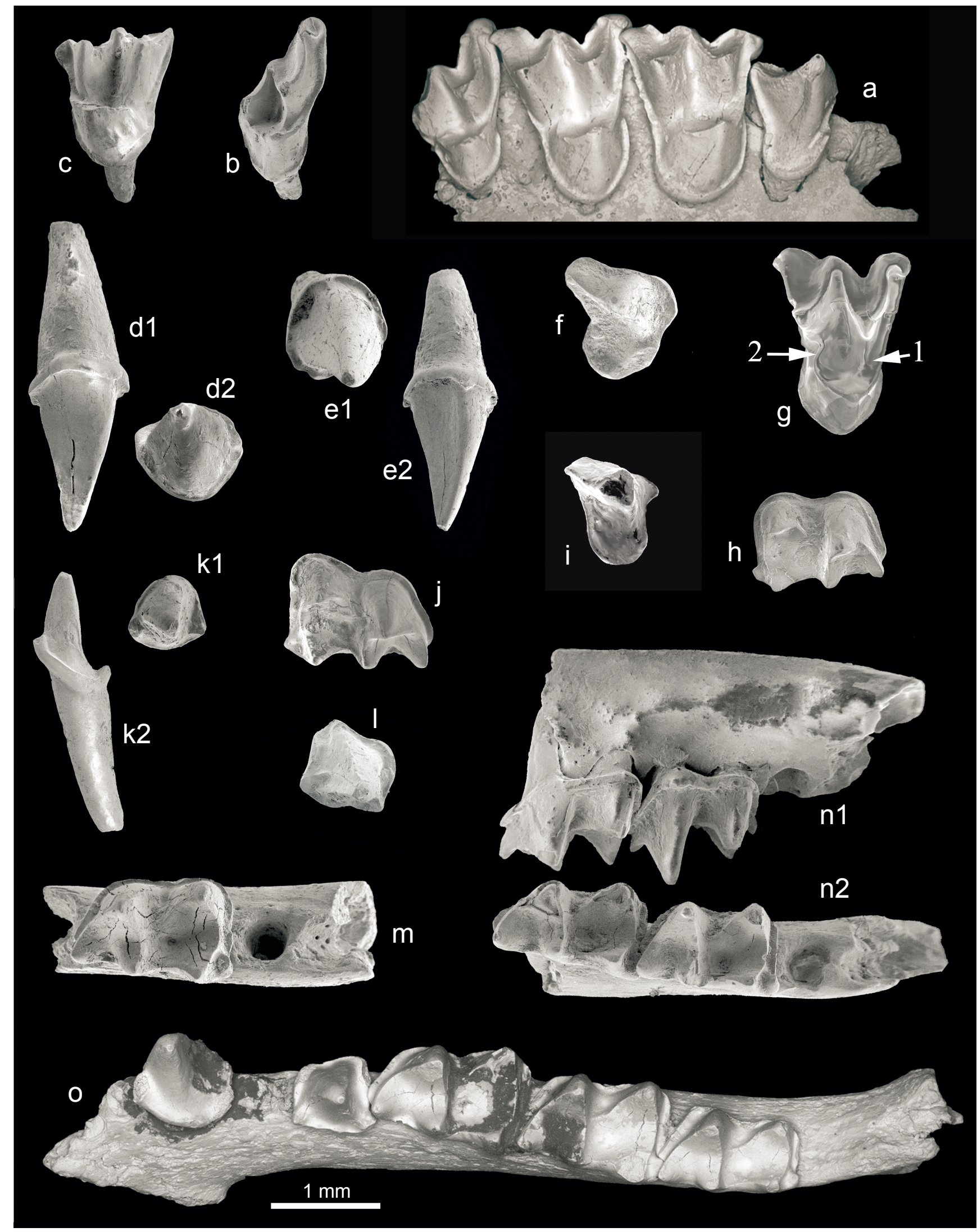

Text-fig. 8. a, o - Miostrellus petersbuchensis, Petersbuch 28: a - NMA P28/0335, right maxillary bone with P4-M3, holotype, ventral view; o - NMA P28/0449, right mnd with C inf., p4-m3, occlusal view; b-f, $k, n-M$. cf. noctuloides: b - BSP 1962 XIX 4198, right M3, Erkertshofen 1, occlusal view; c - BSP 1962 XIX 4195, left M1, Erkertshofen 1, occlusal view; d - PCMRCh4, left C sup., Petersbuch 2, lingual (d1) and occlusal (d2) views; e - BSP 1974 XIV 1204, left C sup., Erkertshofen 2, occlusal (e1) and lingual (e2) views; f - PCMRCh23, right P4, Petersbuch 2, occlusal view; k - BSP 1974 XIV 1200, right C inf., Erkertshofen 2, occlusal view; n - BSP 1962 XIX 4192, right mnd with m1-2; Erkertshofen 1, lateral (n1) and occlusal (n2) views; g - Eptesicus cf. aurelianensis, BSP 1962 XIX 4197, right M2, Erkertshofen 1, occlusal view; h, i - cf. Miostrellus sp., Erkertshofen 2: h - BSP 1974 XIV 1203, left m1, occlusal view; i - BSP 1974 XIV 1207, right P4; j, l, m - Miostrellus cf. petersbuchensis, Petersbuch 2 , occlusal views: j - PCMRCh5, left m1; l - PCMRCh7, left p4; $\mathrm{m}$ - PCMRCh6, right mnd with $\mathrm{m} 1$. 


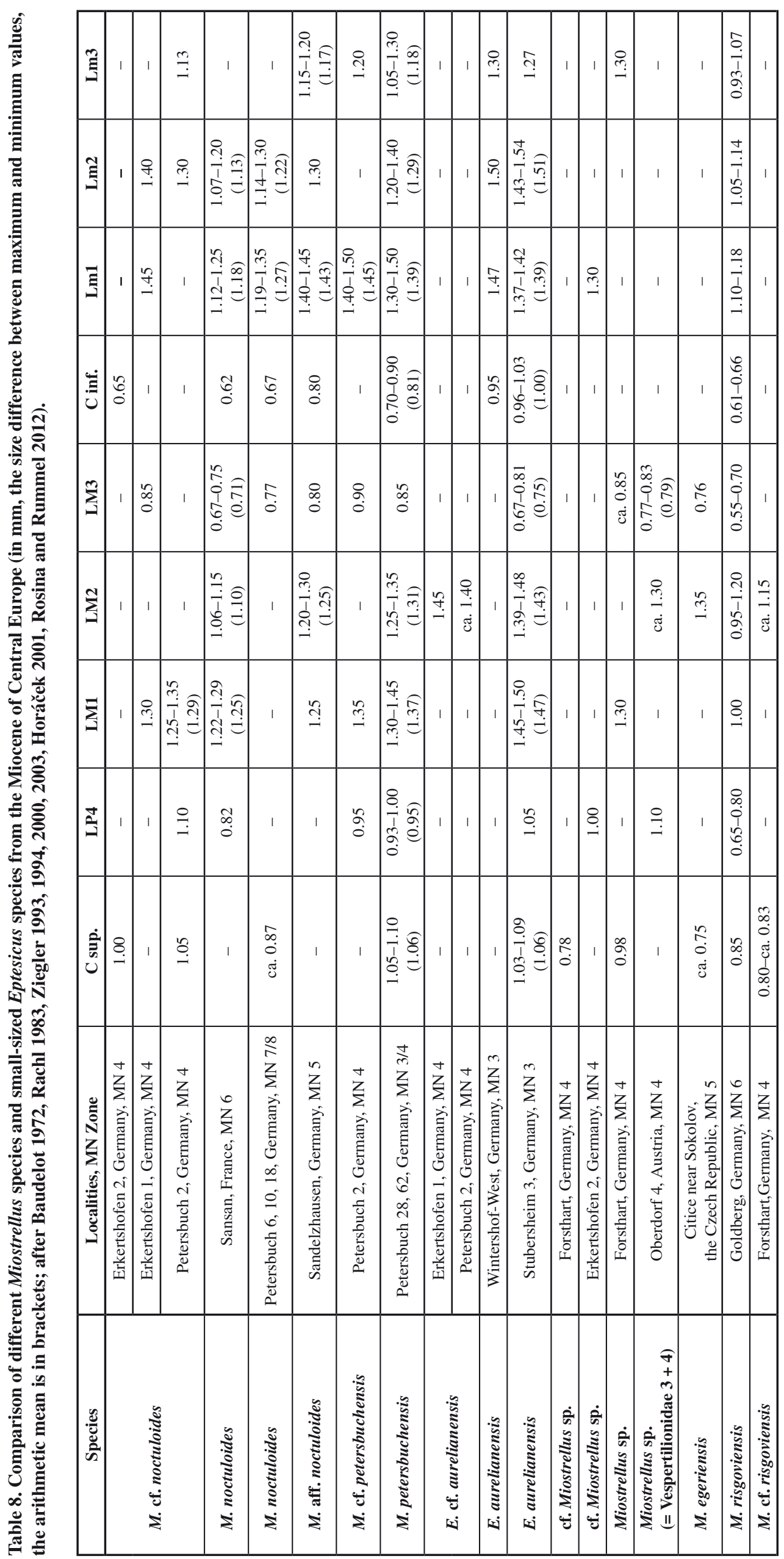


the same-sized M. petersbuchensis and M. egeriensis (Tab. 8) in having paralophs (Text-fig. 8b, c; Rosina and Rummel 2012: 471, fig. 5A, Horáček 2001: 140, fig. 24). The M1 from Erkertshofen 1 is very similar to that of $M$. noctuloides from Sansan in size and in having a small hypocone (compare with Baudelot 1972: 369, pl. II, figs 10, 11; p. 57, fig. 21).

The M3 (specimen BSP 1962 XIX 4198; Text-fig. 8c) differs from Miostrellus sp. from Forsthart (specimen BSP 1959 XXVII-Ch3, MN 4, Germany) in having a less developed cingulum in the protocone area (compare with Rosina and Rummel 2017: 229, fig. 1e, g). The crown of M3 from Erkertshofen 1 is less compressed in the anteroposterior direction in comparison with E. aurelianensis (specimen SMNS 45744 E1; Ziegler 1994: 113, pl. 5, fig. 4). The isolated third upper molars from Stubersheim 3 are more compressed anteroposteriorly but wider than the M3 from Erkertshofen 1, thus, their crowns morphologically are very similar to those of Plecotus or Corynorhinus (Ziegler 1994: 113, pl. 5, fig. 7). The M3 from Erkertshofen 1 is very similar to M3 of $M$. aff. noctuloides from Sandelzhausen (specimen BSP 1959 II 7770, MN 5, Germany; Ziegler 2000: 127, pl. 10, fig. 122) in having a well-developed paraloph and less development of the metacone (Text-fig. 8b). The M3 of the nominative M. noctuloides could probably also have a paraloph because this morphological trait is present in the M1 and M2 crowns of this species (Baudelot 1972: 369, pl. II, figs 10, 11). Nevertheless, the M3 from Erkertshofen 1 is somewhat larger than M. noctuloides from Sansan and from Petersbuch 6 (specimen P6-01046, MN 7/8, Germany; Ziegler 2003: 462, fig. 3(3)).

The crowns of the upper canines from Erkertshofen 2 and Petersbuch 2 (specimens BSP 1974 XIV 1204 and PCMRCh4) are triangular in cross-section, with a welldeveloped cingulum, and without a lingual talon. The posterior and lingual crests are also well-developed, while the buccal crest is less pronounced, and the anterobuccal ridge is rounded (Text-fig. $8 \mathrm{~d}$, e). The shape of the crowns of the upper canines from Erkertshofen 2 and Petersbuch 2 correspond most closely to Menu's type A (Menu 1985: 92, fig. 7). The teeth are very similar to M. risgoviensis (Rachl 1983: 229, fig. 70a, c) but evidently larger in size (Tab. 8). They differ from E. aurelianensis from Stubersheim 3 (SMNS 45744 E1; Ziegler 1994: 113, pl. 5, fig. 4) in having a less-developed lingual crest which is located more anteriorly (Text-fig. 8d, e). Thus, the upper canines from Erkertshofen 2 and Petersbuch 2 morphologically are most similar to $M$. aff. noctuloides from Sandelzhausen (specimen BSP 1959 II 7754; Ziegler 2000: 127, pl. 10, fig. 118).

The crown of the P4 from Petersbuch 2 (specimen PCMRCh23) is compressed in the anteroposterior direction which differentiates it from that of Myotis (Text-fig. 8f). In contrast to Corynorhinus the P4 from Petersbuch 2 does not show a strongly-developed lingual talon (compare with Rosina et al. 2019: 4, fig. 3B). Nevertheless, a small lingual talon is present. This differentiates the P4 from Petersbuch 2 from that of the modern Plecotus (Rosina et al. 2019: 4, fig. 3I). It also differs from the P4 of the Miocene Plecotus species in being more compact in form and in being narrower in width (Text-fig. 8f; compare with Rosina et al. 2019: 4, fig. 3A). Considering the shape, the P4 from Petersbuch 2 is most similar to Eptesicus or Miostrellus. It differs from M. petersbuchensis (Rosina and Rummel 2012: 471, fig. 5A) and from $M$. aff. noctuloides from Sandelzhausen (specimen BSP 1959 II 7756; Ziegler 2000: 127, pl. 10, fig. 119) in having a distinct anterolingual cuspid. The P4 from Petersbuch 2 is similar in size to the P4 of E. aurelianensis which also has an anterolingual cuspid (Ziegler 1994: 112). However, the $\mathrm{P} 4$ of $E$. aurelianensis is wider than the specimen from Petersbuch 2 (see the measurements of specimen SMNS 45744 F1 in Ziegler 1994: 112). The crown shape of the P4 from Petersbuch 2 is very similar to those of $M$. risgoviensis and $M$. noctuloides, but somewhat larger than both of them (Tab. 8; compare with specimen Sa. 13.616 from Sansan in Baudelot 1972: 57, fig. 21).

In contrast to Myotis, the crown of the lower canine from Erkertshofen 2 (specimen BSP 1974 XIV 1200) is somewhat compressed in the anteroposterior direction and the cingulid has a higher anterolingual cuspid (Text-fig. 8k). Morphologically it is close to Menu's type B1 (Menu 1985: 98, fig. 12). The lower canine from Erkertshofen 2 is smaller in size than the lower canines of Miostrellus petersbuchensis (Tab. 8; compare with Rosina and Rummel 2012: 471, fig. $5 \mathrm{E})$. It is morphologically most similar to $M$. noctuloides from Sansan and M. noctuloides from Petersbuch 6 (Tab. 8; Baudelot 1972: 54, fig. 18; specimen NMA P6-1045; Ziegler 2003: 462, fig. 3(2)).

The lower molars from Erkertshofen 1 and Petersbuch 2 are myotodont. The trigonids of the $\mathrm{m} 2$ on the mandible fragments (specimens BSP 1962 XIX 4192, BSP 1962 XIX 4193) are compressed, the $m 1$ paralophids are curved while their talonids are much wider than the trigonids (Text-fig. $8 n$ ). All these traits differentiate the lower molars from Erkertshofen 1 and Petersbuch 2 from the molars of Myotis. The m3 talonid of the specimen BSP 1977 XXII 4800 from Petersbuch 2 is markedly reduced. Morphologically the lower molars from Erkertshofen 1 and Petersbuch 2 are similar to Miostrellus risgoviensis (Text-fig. 8N; Rachl 1983: 233, fig. 71), but significantly larger in size (Tab. 8). They are also larger than $M$. noctuloides and M. petersbuchensis while smaller than E. aurelianensis (Tab. 8). Thus, the lower molars from Erkertshofen 1 and Petersbuch 2 are most similar to $M$. aff. noctuloides from Sandelzhausen in morphology and size (Tab. 8, specimens BSP 1959 II 7728, 7729; Ziegler 2000: 127, pl. 10, fig. 119).

\section{Miostrellus cf. petersbuchensis Rosina et Rummel, 2012 Text-fig. 8j, 1, m}

Material and measurements. Petersbuch 2: PCMRCh5, left m1, $1.50 \times 0.85 \times 0.95 ;$ PCMRCh6, right mnd with $\mathrm{m} 1,1.40 \times 0.90 \times 0.95 ; \mathrm{PCMRCh} 7$, left p $4,0.75 \times$ 0.70; PCMRCh87, right m3, $1.20 \times 0.70 \times 0.65 ; \mathrm{BSP} 1979$ XXII 5365, left M1, $1.35 \times 1.60$; BSP 1979 XXII a, left M1, $1.35 \times 1.65$; BSP 1979 XXII 5367, left M3, $0.90 \times 1.70$; BSP 1980 XXII 4805, left P4, $0.95 \times 0.90$.

Description and comparison. The M1 from Petersbuch 2 (specimens BSP 1979 XXII 5365 and BSP 1979 XXII a) have neither paraloph nor paraconule. The weak metaloph merges with the postprotocrista and disappears at the base of the metacone and thus, the trigon basin is closed. The hypocone is almost absent. The M3 crown from Petersbuch 2 (specimen BSP 1979 XXII 5367) is somewhat compressed in the anteroposterior direction, the metacone is reduced, 
the paraloph is weak and the paraconule is absent. The P4 crown (specimen BSP 1980 XXII 4805) is also compressed in the anteroposterior direction, its lingual talon is moderately developed and the anterolingual cuspid is absent. The upper molars from Petersbuch 2 are distinguished from those of Myotis in having of a well-developed cingulum, a compact crown of upper molars and a lack of conules and hypocones. Morphologically they are most similar to Miostrellus petersbuchensis and also in size (Tab. 8).

The p4 crown from Petersbuch 2 (specimen PCMRCh7) is triangular in occlusal view and has well-developed anterolingual and posterolingual cuspules (Text-fig. 81). It differs from $M$. noctuloides from Sansan and Petersbuch 6 (compare with specimens NMA P6-01045, NMA P1000591; Ziegler 2003: 462, fig. 3(2)) in being larger (Tab. 8). Morphologically and also in size the p4 from Petersbuch 2 is most similar to that of M. petersbuchensis (Tab. 8).

The $\mathrm{m} 1$ paralophids from Petersbuch 2 (specimens PCMRCh5, PCMRCh6) are somewhat curved while the trigonids of the crowns are very wide (Text-fig. 8j, m) which differentiate these teeth from those of Myotis. Nevertheless, the $\mathrm{m} 1$ paralophids are significantly less curved than those of Plecotus. These morphological traits of the crowns, together with their myotodonty, supports their possibly connection to either Eptesicus or Miostrellus. The m3 crown from Petersbuch 2 (specimen PCMRCh87) differs from that of Myotis in having a somewhat reduced talonid, an elongated and curved paralophid and a narrow trigonid. The lower molars are smaller than those of E. aurelianensis but larger than $M$. risgoviensis and $M$. noctuloides (Tab. 8). Therefore they morphologically correspond well with $M$. petersbuchensis (Rosina and Rummel 2012: 471, fig. 5E, F).

\section{cf. Miostrellus sp. \\ Text-fig. 8h, i}

Material. Erkertshofen 2: BSP 1974 XIV 1207, right P4, $1.00 \times 1.00$; BSP 1974 XIV 1203, left m1, $1.30 \times$ $0.75 \times 0.80$.

Description and comparison. The P4 crown from Erkertshofen 2 (specimen BSP 1974 XIV 1207) is compressed in the anteroposterior direction with a weakly pronounced anterolingual cuspid on the cingulum but without a posterolingual talon (Text-fig. 8i). All these features differentiate the P4 from Erkertshofen 2 from those of Myotis and Plecotus. It differs from Submyotodon petersbuchensis (Ziegler 2003: 478, fig. 6(7)) in being larger (Ziegler 2003: 480-481, tab. 8), in having a less developed anterolingual cuspid on the cingulum and in lacking the posterolingual talon. The P4 from Erkertshofen 2 morphologically is similar to $M$. aff. noctuloides from Sandelzhausen (specimen BSP 1959 II 7756; Ziegler 2000: 127, pl. 10, fig. 119) but differs in being larger (Tab. 8) and in having a small anterolingual cuspid on the cingulum. On the other hand, it is similar in size to $M$. petersbuchensis (Tab. 8) which, however, has no anterolingual cuspid of the cingulum (compare with Rosina and Rummel 2012: 471, fig. 5A). The $\mathrm{m} 1$ from Erkertshofen 2 (specimen BSP 1974 XIV 1203) shows an elongated, but somewhat curved, paralophid and a wide trigonid, that indicate it most likely does not belong to Myotis, but to Miostrellus. It is larger than M. risgoviensis, but smaller than both the

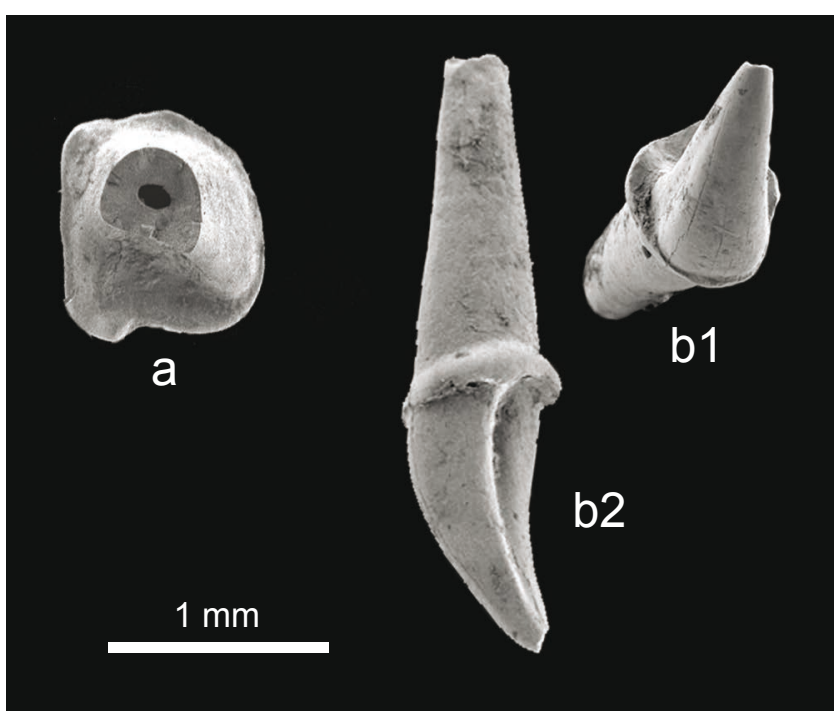

Text-fig. 9. a - Vespertilionidae indet., PCMRCh24, right C inf., Petersbuch 2, occlusal view; b - Chiroptera indet., BSP 1974 XIV 1206, left C sup., Erkertshofen 2, (b1) occlusal view, (b2) lingual view.

M. petersbuchensis and E. aurelianensis and closest in size to M. noctuloides from Sansan (Tab. 8).

\section{Vespertilionidae indet. Text-fig. 9a}

Material and measurements. Petersbuch 2: PCMRCh24, right C inf., $1.05 \times 1.00$.

Description and comparison. The top of this fossil tooth is broken off. However, the well-developed cingulum of the crown and a relatively high anterolingual cuspid of the crown cingulum (Text-fig. 9a) indicate that the specimen belongs to a small representative of the family Vespertilionidae.

\section{Chiroptera indet. Text-fig. 9b}

Materia 1. Erkertshofen 2: BSP 1974 XIV 1205, left C sup.; BSP 1974 XIV 1206, right C sup.

Description and comparison. As in many microchiropteran bats, the crowns of the upper canines from Erkertshofen 2 have a well-developed cingulum. The crowns are crescent shaped in a forward direction, as in rhinolophids or molossids. However, the crowns are rounded in crosssection with a well-developed cingulum, which is thinner on the anterior side (Text-fig. 9b) and thus, relate them to the vespertilionid bats.

\section{Discussion}

Faunal compositions of bat assemblages of the early Miocene sites of Erkertshofen 1, Erkertshofen 2 and Petersbuch 2: palaeoecological and taphonomic remarks

The fossil bat assemblages of Erkertshofen 1, Erkertshofen 2 and Petersbuch 2 originate from the karst fissure 
Table 9. The taxonomical compositions of bat assemblages from Petersbuch 2, Erkertshofen 1 and Erkertshofen 2 ( $\mathbf{N}$ - number of specimens, $\%$ - frequency in percentages).

\begin{tabular}{|c|c|c|c|c|c|c|}
\hline \multirow{2}{*}{ Taxon } & \multicolumn{2}{|c|}{ Petersbuch 2} & \multicolumn{2}{|c|}{ Erkertshofen 2} & \multicolumn{2}{|c|}{ Erkertshofen 1} \\
\hline & $\mathbf{N}$ & $\%$ & $\mathbf{N}$ & $\%$ & $\mathbf{N}$ & $\%$ \\
\hline Chiroptera indet. & - & - & 2 & 1.71 & - & - \\
\hline RHINOLOPHIDAE & 364 & 57.78 & 91 & 77.78 & 48 & 81.35 \\
\hline Rhinolophus aff. lemanensis & 148 & 40.66 & 48 & 52.75 & 23 & 47.92 \\
\hline R. cf. delphinensis & 2 & 0.55 & 3 & 3.3 & 10 & 20.83 \\
\hline R. dehmi & 198 & 54.39 & 39 & 42.86 & 14 & 29.17 \\
\hline R. grivensis & 16 & 4.39 & 1 & 1.1 & 1 & 2.08 \\
\hline VESPERTILIONIDAE & 197 & 31.27 & 24 & 20.51 & 8 & 13.56 \\
\hline Hanakia agadjaniani & 172 & 87.31 & 14 & 53.33 & - & - \\
\hline$H$. aff. antiqus & 3 & 1.52 & 1 & 4.17 & 1 & 12.5 \\
\hline Miostrellus cf. petersbuchensis & 8 & 4.06 & - & - & - & - \\
\hline Myotis cf. reductus & 2 & 1.01 & 5 & 20.83 & 1 & 12.5 \\
\hline cf. Myotis sp. & 2 & 1.01 & - & - & - & - \\
\hline Plecotus cf. atavus & 1 & 0.51 & - & - & 1 & 12.5 \\
\hline Eptesicus cf. aurelianensis & 1 & 0.51 & - & - & 1 & 12.5 \\
\hline Miostrellus cf. noctuloides & 7 & 3.55 & 2 & 8.33 & 4 & 50 \\
\hline cf. Miostrellus sp. & - & - & 2 & 8.33 & - & - \\
\hline Vespertilionidae indet. & 1 & 0.51 & - & - & - & - \\
\hline MEGADERMATIDAE & 69 & 10.95 & - & - & 3 & 5.08 \\
\hline Megaderma franconica & 69 & 100 & - & - & 3 & 100 \\
\hline Total & 630 & 100 & 117 & 100 & 59 & 100 \\
\hline
\end{tabular}

filling deposits in the Jurassic limestone in southern Germany. As a rule, fossil bat faunas from karst sites demonstrate significantly greater taxonomic diversity compared to nonkarst faunas (Rosina and Sinitsa 2014, Rosina and Rummel 2017 etc.). The difference between karstic and non-karstic sites in abundance and composition of fossil bat assemblages is evidently caused by taphonomic reasons, including habitat preferences of different bat species. The karst cavities represent favorite bat roosts and many bat species represent those which were strict cave-dwellers throughout all stages of their annual life cycle. The environment of karst landscapes in the past, as well as in the present, provided opportunities for bat bone accumulation in the karst deposits as the result of both the natural death of animals occurring in large colonies inhabiting karst cavities and from avian pellets. This is demonstrated by the numerous Neogene karst sites of Europe, especially by the Miocene sites of Petersbuch in Germany (Ziegler 2003, Rosina and Rummel 2012, 2017). Accordingly, the mammal assemblages of Erkertshofen 1, Erkertshofen 2 and Petersbuch 2 are rich in bat fossils, belonging to at least 12 different species belonging to Vespertilionidae, Rhinolophidae and Megadermatidae (hereafter see Tab. 9). The rhinolophids are the most numerous accounting for at least $50 \%$ of all bat remains, followed by vespertilionids also common in all sites (no more than $30 \%$ ), while the contribution of megadermatids is much lower (no more than $11 \%$ ). No megadermatids were found in the fauna of Erkertshofen 2, which nevertheless is relatively rich in bat bone specimens.

The vespertilionid bats were abundant in all three oryctocenoses being represented with at least eight taxa, of which Hanakia agadjaniani was the most common and accounted for up to $90 \%$ of the total vespertilionids material in Petersbuch 2 . This relatively large vespertilionid bat could be an attractive prey species for avian predators, whose pellets could be a source for the bat bones accumulated in these deposits. Moreover, remains of Hanakia agadjaniani are rather common in other early Miocene karst sites of Petersbuch (e.g. Rosina and Rummel 2012). This suggests that this species formed large maternity and/or hibernation colonies and used cavities or crevices for shelter. It is noteworthy that the rare remains of another species $H$. aff. antiquus, which morphologically is very close to $H$. agadjaniani but noticeably smaller in size, were found in all three faunas. These two Hanakia species are distinguished by the structure of the crowns of the upper molars (see details above) which excludes any possibility of ascribing the difference between them to sexual dimorphism within a single taxon.

Some remains of Miostrellus cf. petersbuchensis, known from the early Miocene sites of Petersbuch (Rosina and Rummel 2012), were found in Petersbuch 2. The rare fossil remains of this species were also discovered in the middle Miocene non-karstic site Hasznos of Hungary (Rosina et al. 2015). Another form of $M$. cf. noctuloides is more representative of the faunas of Erkertshofen 1 and Erkertshofen 2 (Tab. 9). This form is related to the species M. noctuloides, which is quite common in the middle and late Miocene faunas of Europe (e.g. Baudelot 1972, Ziegler 2000, 2003). Up to now the nominative species E. aurelianensis had been discovered only from the early Miocene bat assemblages of Wintershof-West and Stubersheim 3 (Ziegler 1993, 1994). The only two fragments attributed to Eptesicus cf. aurelianensis were found in Erkertshofen 1 and Petersbuch 2 (Tab. 9). The other vespertilionid fossils 
of Myotis cf. reductus found in the bat assemblages of Erkertshofen 1 and Erkertshofen 2 are quite common in the Miocene bat faunas of Germany (e.g. Ziegler 2003, Rosina and Rummel 2012). Nevertheless, it was represented by a few remains of this species in Petersbuch 2 only (Tab. 9).

It is interesting that each of the sites of Petersbuch 2, Erkertshofen 1 and Erkertshofen 2 include remains of four different species of rhinolophid bats (Text-fig. 10, Tab. 9). Usually only two rhinolophid species are present in many Neogene bat assemblages of Europe (e.g. Ziegler 2003, Rosina and Rummel 2012). There are two larger and two smaller rhinolophid species which were found together in the bat assemblages of Petersbuch 2, Erkertshofen 1 and Erkertshofen 2 (Tab. 9). The majority of the rhinolophid remains (up to $53 \%$ ) belongs to the large Neogene species of Rhinolophus aff. lemanensis, which was first described from the locality of Saint Gérand (MN 2a; Revilliod 1920). It is a very typical species for the early Miocene bat faunas of Europe (e.g. Ziegler 1993, 1994, 1998, Rosina and Rummel 2012). A somewhat smaller rhinolophid, $R$. dehmi, occupied the second position relative to number (no less than $30 \%$ ). This species is known only from the early Miocene of Germany (Ziegler 1993). In addition, rare remains of $R$. grivensis (up to $4 \%$ in Petersbuch 2 ) and a larger form, $R$. cf. delphinensis (up to $20 \%$ in Erkertshofen 1; Tab. 9) were also attributed to the assemblage, both these taxa are more characteristic of the later Miocene faunas of Europe. Such a high diversity of rhinolophids is typical for modern tropical forest and subtropical palaeotropic faunas (e.g. Struebig et al. 2012, Tu et. al. 2016). Thus, the taxonomic composition of bat assemblages from Petersbuch 2, Erkertshofen 1 and Erkertshofen 2 characterises a tropical or subtropical type of palaeobiota in the early Miocene in South Germany.
Only a few fossils of the megadermatid bat, Megaderma franconica, were found at Petersbuch 2 and Erkertshofen 1. The number does not reach $20 \%$ in either location (Tab. 9). Until now, this fossil species was known only from the Miocene of Germany (e.g. Ziegler 1993, 2003, Rosina and Rummel 2012). The presence of the numerous fossils of fairly large-sized species of bats, such as $R$. aff. lemanensis, $H$. agadjaniani and $M$. franconica in Erkertshofen 1, Erkertshofen 2 and Petersbuch 2 suggests a colonial lifestyle in these species. In modern times, the colonial species of large-sized bats often become prey for owls, hawks and falcons, whose pellets can be the origin of the bat bone aggregations in karst deposits (see references in Rosina and Sinitsa 2014). Obviously, the natural death of bats from the colonies inhabiting palaeokarst cavities is also a reason for the accumulation of bat bones in the Miocene deposits. This is especially clearly indicated by the presence of a large number of remains of significantly smaller-sized bats, such as $R$. dehmi and Miostrellus s. str. in the same assemblages. Small-sized modern bats are much less likely to become prey of avian predators (see references in Rosina and Sinitsa 2014). Moreover, the bat bone remains do not bear obvious traces of the effects of digestive enzymes. This lends even more support to the premise that their accumulation is most likely a result of the natural death of the animals inhabiting the bat colonies. However, it is impossible to exclude the possibility that the bat bone accumulation also resulted from the pellets of birds of prey.

\section{Biostratigraphic correlation and comparison of the bat faunas from Erkertshofen 1, Erkertshofen 2 and Petersbuch 2 with other Miocene bat faunas of Europe}

Bats are one of the most numerous and widely distributed order of modern mammals, the highest diversity of taxa is

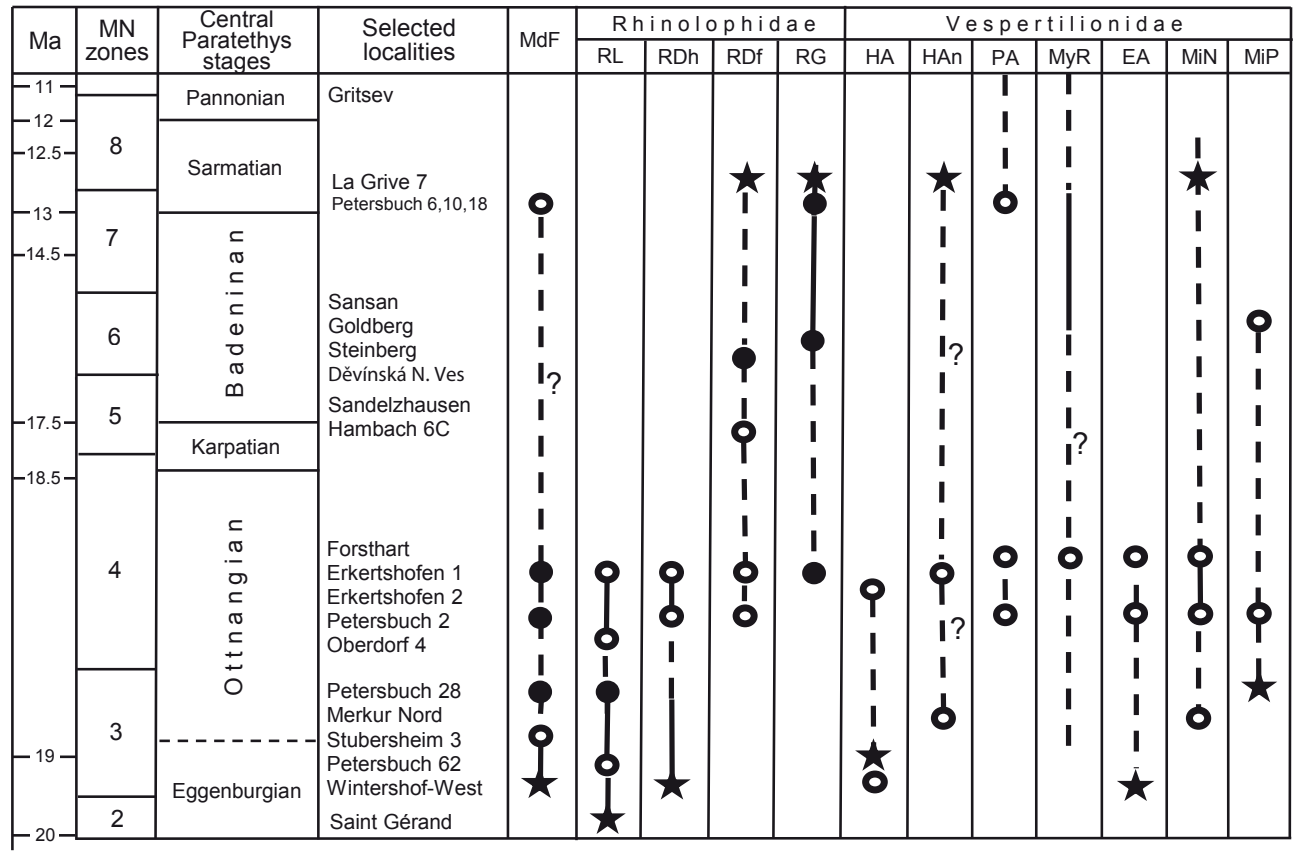

MdF - Megaderma franconica $\mathrm{RL}$ - Rhinolophus lemanensis $\mathrm{RDh}$ - Rhinolophus dehmi RDf - Rhinolophus delphinensis RG - Rhinolophus grivensis HA - Hanakia agadjaniani HAn - Hanakia antiquus PA - Plecotus atavus MyR - Myotis reductus EA - Eptesicus aurelianensis MiN - Miostrellus noctuloides MiP - Miostrellus petersbuchensis

apparent record

0 aff. or cf.

$\star$ record from the type locality

? - - the estimated biostratigraphic range of a taxon

- the biostratigraphic range of a taxon confirmed by fossil finds

Text-fig. 10. The chronostratigraphic position of the sites of Erkertshofen 1, Erkertshofen 2 and Petersbuch 2 and the biochronology of some European Neogene bats in Central Europe (species distribution after Revilliod 1920, Zapfe 1950, Rachl 1983, Ziegler 1993, 1994, 1998, 2000, 2003, Horáček 2001, Mörs 2002, Ginsburg and Mein 2012, Rosina and Rummel 2012, 2017, Rasser et al. 2013, Rosina et al. 2019). Miocene time scale after Steininger 1999, MN zones modified after Jones 1999, Rögl 1999. 
observed in tropical and subtropical regions (Findley 1993, Struebig et al. 2012). The fossil records of bats are also well represented, both taxonomically and biostratigraphically (e.g. Eiting and Gunnell 2009). Thus, bat remains are very numerous and diverse in many of the Neogene mammal faunas (e.g. Horáček 2001, Ziegler 2003, Rosina and Rummel 2012 etc.). However, bats still have a very limited use as stratigraphic indicators in biostratigraphic studies. This is mostly due to the limited information on the phylogenetic morphoclines of particular clades, palaeoecology and life history traits of fossil bats, as well as due to the absence of such information for many modern species, despite much research in these scientific areas (e.g. Kingston et al. 2003, Benda et al. 2010 etc.). Recent bats are the only mammals with active flight, which determines not only their wide distribution, but often the difficulty of studying their biology and ecology. Such a lack of information on the ecology of modern species increases the difficulties of palaeoecological reconstructions of the fossil communities of bats, making them unsuitable for biostratigraphic studies. Compared to rodents and insectivores, bats are much rarer prey of modern birds of prey and, accordingly, the bone remains of bats are much less likely to be in the pellet material and thus subsequently be incorporated into the fossil taphocoenoses. Together this makes it difficult to find unambiguous stratigraphic indicators among the bats that could be used for biostratigraphic studies. However, at times analysis of the taxonomic composition of the fossil bat aggregations allows one to confidently argue the biostratigraphic correlations of different sites and to estimate their age.

The early Miocene bat faunas from Petersbuch 2, Erkertshofen 1 and Erkertshofen 2 are markedly similar to each other in the taxonomic composition and the species ratio (Tab. 9). The $R$. aff. lemanensis and $R$. dehmi are the most abundant in all three faunal assemblages. These early Miocene bat species have so far only been found in the localities whose age has been estimated to be not younger than MN 4 (e.g. Oberdorf 4, Petersbuch 28, Petersbuch 62; Text-fig. 10). However, the faunas from Petersbuch 2 and Erkertshofen 2 also include $H$. agadjaniani, which until now is confidently known from early Miocene locations, whose age correlates with MN 3 (e.g. Wintershof-West, Stubersheim 3). The presence of abundant remains of $R$. cf. delphinensis and Miostrellus cf. noctuloides, the typical elements of the middle and the late Miocene bat faunas of Europe (e.g. MN 7-8, Sansan, France; Ginsburg and Mein 2012; MN 5, Casetón 1A and 2B, Spain; Sevilla 2002; MN 7-8, Petersbuch 6, 10, 18, Germany; Ziegler 2003) in Erkertshofen 1 may indicate a somewhat younger age of the mammal fauna from this site than either Erkertshofen 2 and Petersbuch 2. Such an estimation of age correlation between the Erkertshofen 1, Erkertshofen 2 and Petersbuch 2 has also been suggested by other small mammals (Fahlbusch and Ziegler 1986, Roth 1989).

The taxonomic composition of the bat faunas of Petersbuch 2, Erkertshofen 1 and Erkertshofen 2 sites are similar to those of Petersbuch 28, Petersbuch 62 and Wintershof-West (Text-fig. 10), yet differ in the presence of Plecotus cf. atavus and $H$. aff. antiquus, the taxa more typical of the middle Miocene bat faunas (e.g. Petersbuch 6, MN 7/8; Ziegler 2003). The presence in these sites of $R$. aff. lemanensis and
R. dehmi on the one hand, and Plecotus cf. atavus and $H$. aff. antiquus, on the other hand, suggests an age not younger than MN 4 but not older than MN 3. The Petersbuch 2 site seems to be the oldest of the three sites under study due to the presence of the abundant remains of $R$. aff. lemanensis, $R$. dehmi and $H$. agadjaniani and Miostrellus cf. petersbuchensis, which is absent in both Erkertshofen 1 and Erkertshofen 2 (Tab. 9). Thus, a comprehensive analysis of the bat faunas from sites of similar taphonomic origin allowed estimation of their biostratigraphic correlation. Clearly, analysis of the distribution of the faunal complexes, which includes certain bat taxa, could be useful in biostratigraphic studies. Thus, not only the fossil bat species, but their taxonomical aggregations could act as stratigraphic indicators in such work.

\section{Acknowledgements}

We are very grateful to Dr. Timo Körner and Dr. Alexander Hartwig, Applied Materials Laboratory, University of Augsburg, Germany, for permission and assistance in use of the SEM. We gratefully acknowledge Dr. G. E. Rössner, Bavarian State Collection for Paleontology and Geology, Munich, Germany, for permission to work with the fossil bat collections. We greatly appreciate Mr. Colin Matchett's (USA) corrections of the English text. Special gratitude goes to two anonymous reviewers for careful reviewing of the manuscript and useful comments. This study was supported by the Program of Basic Studies of the Presidium of the Russian Academy of Sciences "Evolution of the Organic World and Planetary Processes".

\section{References}

Abdul Aziz, H., Böhme, M., Rocholl, A., Prieto, J., Wijbrans, J. R., Bachtadse, V., Ulbig, A. (2010): Integrated stratigraphy and ${ }^{40} \mathrm{Ar} /{ }^{39} \mathrm{Ar}$ chronology of the early to middle Miocene Upper Freshwater Molasse in western Bavaria (Germany). - International Journal of Earth Sciences, 99: 1859-1886.

https://doi.org/10.1007/s00531-009-0475-8

Baudelot, S. (1972): Étude des Chiroptères, Insectivores et Rongeurs du Miocène de Sansan (Gers); Thèse de doctorat $[\mathrm{PhD}$ thesis]. - MS, Université Toulouse III, Toulouse, France, 496 pp. (copy in library of The Natural Museum of city of Augsburg)

Benda, P., Lučan, R. K., Obuch, J., Reiter, A., Andreas, M., Bačkor, P., Bohnenstengel, T., Eid, E. K., Ševčík, M., Vallo, P., Amr, Z. S. (2010): Bats (Mammalia: Chiroptera) of the Eastern Mediterranean and Middle East. Part 8. Bats of Jordan: fauna, ecology, echolocation, ectoparasites. - Acta Societatis Zoologicae Bohemicae, 74: $185-353$.

Eiting, T. P., Gunnell, G. F. (2009): Global Completeness of the Bat Fossil Record. - Journal of Mammalian Evolution, 16: 151-173. https://doi.org/10.1007/s10914-009-9118-x

Fahlbusch, V. (1966): Cricetidae (Rodentia, Mammalia) aus der mittelmiocänen Spaltenfüllung Erkertshofen bei Eichstätt. - Mitteilungen der Bayerischen Staatssammlung für Paläontologie und historische Geologie, 6: 109-131. 
Fahlbusch, V., Ziegler, R. (1986): Kleinsäuger-Faunen aus der basalen Oberen Süßwasser-Molasse Niederbayerns. - Zitteliana, 14: 3-80.

Findley, J. S. (1993): Bats: a community perspective. Cambridge University Press, Cambridge, 167 pp.

Gaillard, C. (1899): Mammifères Miocènes nouveaux ou peu connus de La Grive-Saint-Alban (Isère). - Archives du Museum d'histoire naturelle de Lyon, 7: 1-78. https://doi.org/10.5962/bhl.title.86383

Ginsburg, L., Mein, P. (2012): Les Chiroptera de Sansan. - In: Peigné, S., Sen, S. (eds), Mammifères de Sansan. Mémoires du Muséum national d'Histoire naturelle, 203: 13-28.

Heissig, K. (1978): Fossilführende Spaltenfüllungen Süddeutschlands und die Ökologie ihrer oligozänen Huftiere. - Mitteilungen der Bayerischen Staatssammlung für Paläontologie und historische Geologie, 18: 237-288.

Heizmann, E. P. J. (1983): Die Gattung Cainotherium (Cainotheriidae) im Orleanium und im Astaracium Süddeutschlands. - Eclogae geologicae Helvetiae, 76: 781-825.

Horáček, I. (2001): On the early history of vespertilionid bats in Europe: the Lower Miocene record from the Bohemian Massif. - Lynx (Praha), n. s., 32: 123-154.

Jones, R. W. (1999): Marine invertebrate (chiefly foraminiferal) evidence for the palaeogeography of the Oligocene-Miocene of western Eurasia, and consequences for terrestrial vertebrate migration. - In: Agustí, J., Rook, L., Andrews P. (eds), The Evolution of Neogene Terrestrial Ecosystems in Europe. Cambridge University Press, Cambridge, pp. 274-308. https://doi.org/10.1017/CBO9780511542329.014

Kälin, D., Kempf, O. (2009): High-resolution stratigraphy from the continental record of the Middle Miocene Northern Alpine Foreland Basin of Switzerland. - Neues Jahrbuch für Geologie und Paläontologie, Abhandlungen, 254: 177-235.

https://doi.org/10.1127/0077-7749/2009/0010

Kingston, T., Francis, C. M., Akbar Z., Kunz, T. H. (2003): Species richness in an insectivorous bat assemblage from Malaysia. - Journal of Tropical Ecology, 19: 67-79. https://doi.org/10.1017/S0266467403003080

Mein, P. (1964): Chiroptera (Miocène) de Lissieu (Rhône). - Comptes Rendus du 89e Congrès national des Sociétés Savantes, Section Sciences, Lyon, pp. 237-253.

Menu, H. (1985): Morphotypes dentaires actuels et fossiles des chiroptères vespertilioninès. Ie partie: étude des morphologies dentaires. - Palaeovertebrata, 15: 71-128.

Miller, G. S. (1907): The Families and Genera of Bats. Smithsonian Institution US National Museum Bulletin, 57: 1-282. https://doi.org/10.5962/bhl.title.55695

Mörs, T. (2002): Biostratigraphy and paleoecology of continental Tertiary vertebrate faunas in the Lower Rhine Embayment (NW-Germany). - Netherlands Journal of Geosciences / Geologie en Mijnbouw, 81(2): 177-183. https://doi.org/10.1017/S0016774600022411

Rachl, R. (1983): Die Chiroptera (Mammalia) aus den mittelmiozänen Kalken des Nördlingers Rieses (Süddeutschland); Ph.D. Thesis. - MS, Ludwig-Maximilians-Universität München, Munich, Germany, 284 pp. (copy in library of The Natural Museum of city of Augsburg).
Rasser, M., Bechly, G., Böttcher, R., Ebner, M., Heizmann, E., Höldtke, O., Joachim, C., Kern, A., Kovar-Eder, J., Nebelsick, J., Roth-Nebelsick, A., Schoch, R., Schweigert, G., Ziegler, R. (2013): The Randeck Maar: Palaeoenvironment and habitat differentiation of a Miocene lacustrine system. - Palaeogeography, Palaeoclimatology, Palaeoecology, 392: 426-453.

https://doi.org/10.1016/j.palaeo.2013.09.025

Revilliod, P. (1920): Contribution à l'étude des chiroptères des terrains tertiaires. 2e partie. - Mémoires de la Société Paléontologique Suisse, 44: 63-129.

Rosina, V. V. (2015): First Neogene Otonycteris (Chiroptera: Vespertilionidae) from Ukraine: its biostratigraphic and paleogeographic significance. - Palaeovertebrata, 39(1)-e2: 1-13. https://doi.org/10.18563/pv.39.1.e2

Rosina, V. V., Kruskop, S. V., Semenov, Y. (2019): New Late Miocene plecotine bats (Chiroptera, Vespertilionidae: Plecotini) from Gritsev, Ukraine. - Palaeovertebrata, 42(1)-e2: 1-13. https://doi.org/10.18563/pv.42.1.e2

Rosina, V. V., Prieto, J., Hír, J., Kordos, L. (2015): First record of bats (Chiroptera, Mammalia) from the Middle Miocene non-karstic site Hasznos (Hungary, Nógrád County). - Acta Chiropterologica, 17(2): 283-292. https://doi.org/10.3161/15081109ACC2015.17.2.004

Rosina, V. V., Rummel, M. (2012): The bats (Chiroptera, Mammalia) from the Early Miocene of Petersbuch (Southern Germany, Bavaria). - Geobios 45(5): 463478. https://doi.org/10.1016/j.geobios.2011.10.015

Rosina, V. V., Rummel, M. (2017): The new Early Miocene bat records from the Molasse sites of South Germany. Fossil Imprint, 73(3-4): 227-235. https://doi.org/10.2478/if-2017-0013

Rosina, V. V., Sinitsa, M. V. (2014): Bats (Chiroptera, Mammalia) from the Turolian of Ukraine: phylogenetic and biostratigraphic considerations. - Neues Jahrbuch für Geologie und Paläontologie, Abhandlungen, 272(2): 147-166. https://doi.org/10.1127/0077-7749/2014/0403

Roth, C. (1989): Die Raubtierfauna (Carnivora, Mamm.) der untermiozänen Spaltenfüllung von Erkertshofen 2 bei Eichstätt/Bayern. - Mitteilungen der Bayerischen Staatssammlung für Paläontologie und historische Geologie, 29: 163-205.

Rögl, F. (1999): Mediterranean and Paratethys palaeogeography during the Oligocene and Miocene. - In: Agustí, J., Rook, L., Andrews P. (eds), The Evolution of Neogene Terrestrial Ecosystems in Europe. Cambridge University Press, Cambridge, pp. 8-22. https://doi.org/10.1017/CBO9780511542329.002

Sevilla, P. (2002): Quirópteros fósiles del Aragoniense medio de Casetón (Teruel, España) [Fossil Chiroptera from the middle Aragonian of Casetón (Teruel, Spain)]. - Revista Española de Paleontología, 17(2): 257-268. (in Spanish)

Sigé, B. (1968): Les chiroptères du Miocène inférieur de Bouzigues. I. Étude systématique. - Palaeovertebrata, 1(3): $65-133$. https://doi.org/10.18563/pv.1.3.65-133 
Sigé, B. (1976): Les Megadermatidae (Chiroptera, Mammalia) Miocènes de Beni Mellal, Maroc. - Géologie mediterranéenne, 3(2): 71-86. https://doi.org/10.3406/geolm.1976.963

Simmons, N. B. (2005): Order Chiroptera. - In: Wilson, D. E., Reeder, D. M. (eds), Mammal species of the world: a taxonomic and geographic reference. $3^{\text {rd }}$ ed. Johns Hopkins University Press, Baltimore, Maryland, pp. 312-529.

Steininger, F. (1999): Chronostratigraphy, geochronology and biochronology of the Miocene "European Land Mammal Mega-Zones (ELMMZ)" and the Miocene "Mammal Zones (MN-Zones)". - In: Rössner, G. E., Heissig, K. (eds), The Miocene Land Mammals of Europe. Verlag Dr. Friedrich Pfeil, München, pp. 9-24.

Struebig, M. J., Bozek, M., Hildebrand, J., Rossiter, S. J., Lane, D. J. W. (2012): Bat diversity in the lowland forests of the Heart of Borneo. - Biodiversity Conservation, 21: 3711-3727. https://doi.org/10.1007/s10531-012-0393-0

Tu, V. T., Estók, P., Csorba, G., Son, N. T., Thanh, H. T., Tuan, L. Q., Görföl, T. (2016): Recent remarkable records reveal that Phia Oac-Phia Den Nature Reserve is a priority area for bat conservation in Northern Vietnam. - Journal of Asia-Pacific Biodiversity, 9: 312-322. https://doi.org/10.1016/j.japb.2016.04.007

Viret, J. (1951): Catalogue critique de la faune de mammifères miocènes de La Grive Saint-Alban. Première Partie: Chiroptères, Carnivores, Edentés Pholidotes. Nouvelles Archives du Muséum d'Histoire naturelle de Lyon, 3 : 1-104.
Zapfe, H. (1950): Die Fauna der miozänen Spaltenfüllung von Neudorf an der March (Č.S.R.): Chiroptera. Sitzungsberichte der Österreichischen Akademie der Wissenschaften, Wien, mathematisch-naturwissenschaftliche Klasse, Abt. 1, 159: 51-64.

Ziegler, R. (1993): Die Chiroptera (Mammalia) aus dem Untermiozän von Wintershof-West bei Eichstätt (Bayern). - Mitteilungen der Bayerischen Staatssammlung für Paläontologie und historische Geologie, 33: 119-154.

Ziegler, R. (1994): Die Chiroptera (Mammalia) aus dem Unteromiozän von Stubersheim 3 (Baden-Württemberg). - Münchner Geowissenschaftliche Abhandlungen, A, 26: 97-116.

Ziegler, R. (1998): Wirbeltiere aus dem Unter-Miozän des Lignit-Tagebaues Oberdorf (Weststeirisches Becken, Österreich): 5. Marsupialia, Insectivora und Chiroptera (Mammalia). - Annalen des Naturhistorischen Museums in Wien, A, 99: 43-97.

Ziegler, R. (2000): The bats (Chiroptera, Mammalia) from the Late Oligocene Fissure Fillings Herrlingen 8 and Herrlingen 9 near Ulm (Baden-Württemberg). - Senckenbergiana lethaea, 80(2): 647-683. https://doi.org/10.1007/BF03043368

Ziegler, R. (2003): Bats (Chiroptera, Mammalia) from Middle Miocene karstic fissure fillings of Petersbuch near Eichstätt, Southern Franconian Alb (Bavaria). - Geobios, 36: 447-490. https://doi.org/10.1016/S0016-6995(03)00043-3 